\title{
BONE MORPHOGENETIC PROTEIN 8B PROMOTES THE PROGRESSION OF NON- ALCOHOLIC STEATOHEPATITIS
}

Michele Vacca ${ }^{1,2^{*}}$, Jack Leslie ${ }^{3}$, Samuel Virtue ${ }^{1}$, Brian Y. H. Lam ${ }^{4}$, Olivier Govaere ${ }^{3}$, Dina Tiniakos $^{3,5}$, Sophie Snow ${ }^{6}$, Susan Davies' ${ }^{7}$, Kasparas Petkevicius ${ }^{1}$, Zhen Tong ${ }^{8}$, Vivian Peirce $^{1}$, Mette Juul Nielsen ${ }^{9}$, Zsuzsanna Ament ${ }^{2}$, Wei Li ${ }^{8}$, Tomasz Kostrzewski ${ }^{6}$, Diana Julie Leeming $^{9}$, Vlad Ratziu ${ }^{10}$, Michael ED Allison ${ }^{7}$, Quentin M Anstee ${ }^{3,11}$, Julian L Griffin ${ }^{2,12}$, Fiona Oakley $^{3}$, \& Antonio Vidal-Puig ${ }^{1,13,14^{*}}$

${ }^{1}$ TVP Lab, WT/MRC Institute of Metabolic Science, MRC Metabolic Diseases Unit - Metabolic Research Laboratories, University of Cambridge (UK); ${ }^{2}$ Department of Biochemistry and Cambridge Systems Biology Centre, The Sanger Building, University of Cambridge (UK); ${ }^{3}$ Institute of Cellular Medicine, Faculty of Medical Sciences, Newcastle University, Newcastle upon Tyne (UK); ${ }^{4}$ Yeo Group and Genomics and Transcriptomics Core, WT/MRC Institute of Metabolic Science, University of Cambridge (UK); ${ }^{5}$ Dept of Pathology, Aretaieion Hospital, Medical School, National \& Kapodistrian University of Athens (Greece); ${ }^{6} \mathrm{CN}$ Bio Innovations Ltd, Welwyn Garden City, Hertfordshire, UK; ' 7 Liver Unit, Department of Medicine, Cambridge Biomedical Research Centre, Cambridge University Hospitals (UK); ${ }^{8}$ Department of Medicine, University of Cambridge (UK); ${ }^{9}$ Nordic Bioscience A/S, Herlev (Denmark); ${ }^{10}$ Sorbonne Université, Institute for Cardiometabolism and Nutrition (ICAN), Hôpital Pitié-Salpêtrière, Paris (France). ${ }^{11}$ Newcastle NIHR Biomedical Research Centre, Newcastle upon Tyne Hospitals NHS Foundation Trust, Newcastle upon Tyne (UK); ${ }^{12}$ Biomolecular Medicine, Systems Medicine, Department of Metabolism, Digestion and Reproduction, Imperial College London (UK). ${ }^{13}$ Welcome Trust Sanger Institute, Hinxton (UK). ${ }^{14}$ Cambridge University Nanjing Centre of Technology and Innovation, Jiangbei Area, Nanjing, P.R. of China

\section{* Correspondence: Michele Vacca \& Antonio Vidal-Puig}

TVPlab, MRC Metabolic Diseases Unit - Metabolic Research Laboratories, University of Cambridge - Level 4WT/MRC Institute of Metabolic Science - Box 289, Addenbrooke's Hospital - Hills Road, Cambridge (UK) CB2 0QQ. https://twitter.com/TVPLab

Email addresses: ajv22@medschl.cam.ac.uk; mv400@medschl.cam.ac.uk

Keywords: Non-alcoholic steatohepatitis (NASH), Fibrosis, Hepatic Stellate Cells (HSC), Bone Morphogenetic Proteins, TGF beta. 


\section{ABSTRACT}

Non-alcoholic steatohepatitis (NASH) is characterized by lipotoxicity, inflammation and fibrosis, ultimately leading to end-stage liver disease. The molecular mechanisms promoting $\mathrm{NASH}$ are poorly understood and treatment options are limited. Here, we demonstrate that hepatic expression of Bone Morphogenetic Protein 8B (BMP8B), a member of the TGF $\beta / B M P$ superfamily, increases proportionally to disease stage in patients and animal models of NASH. BMP8B signals via both Smad 2/3 and Smad 1/5/9 branches of the TGF $\beta$ /BMP pathway in hepatic stellate cells (HSC), promoting their pro-inflammatory phenotype. Absence of Bmp8b prevents HSC activation, reduces inflammation, affects the wound healing responses, thereby limiting NASH progression. Specular evidences were obtained in primary human 3D microtissues modelling NASH when challenged with recombinant BMP8. Our data show that BMP8B is a major contributor to NASH progression. Due to the near absence of BMP8B in healthy livers, inhibition of BMP8B might represent a promising new therapeutic avenue for NASH treatment. 
Vacca et al, Bmp8b in NASH

\section{INTRODUCTION}

Non-Alcoholic Fatty Liver Disease (NAFLD) is characterized by excessive accumulation of lipids within the liver. The prevalence of NAFLD is high and progressively increasing, especially in the westernized world ${ }^{1}$, primarily as a consequence of increasing obesity and Metabolic Syndrome prevalence ${ }^{2,3}$. Despite the prognosis of isolated steatosis (non-alcoholic fatty liver, NAFL) being relatively benign per se, exposure to sustained metabolic insults and lipotoxicity contributes to the development of hepatocyte injury, inflammation and liver fibrosis; these histological features define non-alcoholic steatohepatitis (NASH) ${ }^{4,5}$. Moreover, NASH may progress to advanced liver fibrosis/cirrhosis, which is associated with increased mortality and it is a leading cause of liver transplantation ${ }^{1}$. A full understanding of NASH pathophysiology is needed, but is lacking so far. Furthermore, given the limited range of currently approved therapies, the identification of novel targetable pathways able to ameliorate/reverse NASH is a global health priority.

Bone Morphogenetic Proteins (BMPs) are members of the TGF $\beta / B M P$ superfamily. BMPs contribute to liver function in health and disease, having been involved in regulation of iron and lipid metabolism ${ }^{6,7}$, angiogenesis ${ }^{8,9}$, the liver macrophage niche ${ }^{10}$, liver regeneration, and chronic liver disease progression (fibrosis and hepatocellular carcinogenesis) ${ }^{11-13}$. Depending on their liver cell specific roles and their capability to activate Smad 1/5/9 or Smad 2/3 downstream effectors and Smad-independent pathways, TGF $\beta / B M P$ ligands exert counteracting effects on inflammation and compensatory proliferation (promoted by most BMPs, and inhibited by TGFs), HSC activation/Fibrosis (promoted by TGFs and BMP9, and inhibited by BMP7) and cell differentiation (BMPs promote differentiation; TGFs promote EMT) 11,14,15. A comprehensive understanding of the differential action played by each superfamily member in liver disease may thus reveal unique diagnostic and therapeutic opportunities.

Here we studied Bone Morphogenetic Protein 8b (BMP8B), a poorly characterized member of the superfamily, that has been implicated in regulating energy expenditure ${ }^{16,17}$, germ-line cell proliferation and maturation ${ }^{18}$ and cancer ${ }^{19,20}$. The contribution of BMP8B in liver 
pathophysiology is in its infancy and limited to some incidental observations: SNPs in the BMP8B locus have been associated with increased necro-inflammation in patients with viral hepatitis $^{21}$, and upregulation of $B m p 8 b$ can be found in murine models of diet-induced NASH (e.g. choline and folate deficient diet, GSE62362) ${ }^{22}$, and in murine genetic models of NASH and chronic cholestasis (e.g. MDR2KO, or in the IKK $\gamma /$ Nemo KO mice - GSE33161) ${ }^{23,24}$. Currently, the biological consequences of the up-regulation of Bmp8b in these models are unknown also in light of the fact that BMP8B exhibits two unusual characteristics. Firstly, while most BMPs signal via either the ALK4/5/7 (Smad 2/3) or the ALK1/2/3/6 (Smad 1/5/9) branch of the SMAD pathway, BMP8B signals via both ${ }^{17,18}$. Given the key contrasting roles of SMAD branches in regulating liver pathophysiology ${ }^{11}$, we posited that BMP8B may be tuning the TGF $\beta / B M P$ signaling during the wound healing response modulating NASH development and progression. Secondly, $B M P 8 B$ mRNA is almost undetectable in the healthy liver of both mice ${ }^{17}$ and humans (https://www.proteinatlas.org/ENSG00000116985BMP8B/tissue; https://gtexportal.org/home/gene/BMP8B). However, screening publicly available databases, Bmp8b mRNA expression is elevated in experimental models of NAFLD/NASH, with a degree of up-regulation proportional to the entity of damage (Supplementary Table 1).

Here we demonstrate that the expression of BMP8B occurs in hepatocytes and hepatic stellate cells (HSC) in patients and murine models of NASH, proportionally to disease stage. BMP8B activates both Smad $2 / 3$ and $1 / 5 / 9$ in primary murine HSCs, promoting their activation and pro-inflammatory behavior. In diverse in vivo and in vitro models of wound healing and NASH, the absence of Bmp8b prevents HSC activation and/or the activation of inflammatory pathways thus affecting the wound healing responses and preventing NASH progression. Furthermore, using novel human primary 3D micro-tissues challenged with fatty acids to mimic $\mathrm{NASH}^{25}$ and recombinant BMP8, we show that BMP8B is a major contributor to human NASH progression and that our findings can be translated into human NASH pathophysiology. 


\section{RESULTS}

\section{Bmp8b is induced in liver injury and NASH}

Levels of Bmp8b mRNA (Extended Data Figure 1A) and protein (Extended Data Figure 1B \& 1C) expression are really low in the normal mouse liver. However, Bmp8b is upregulated at the mRNA and protein level in both the Western Diet (WD)-induced NASH mouse model, and in the livers of acute or chronic carbon tetrachloride $\left(\mathrm{CCl}_{4}\right)$ injured mice. In diseased livers, Bmp8b is co-localized with Albumin (Alb; Extended Data Figure 1B; produced by hepatocytes) and alpha smooth muscle actin (aSMA; Extended Data Figure 1C; a marker of activated HSC/pericytes) but is not expressed in other liver cell types (Supplementary Figure 1; VE cadherin: vascular cells; CD68: KC/macrophages; K19: biliary and hepatic progenitor cells).

Clinical evidence from two independent cohorts of biopsy-proven NASH patients showed that $B M P 8 B$ mRNA expression in liver biopsies increases with the NASH disease stage (Figure $1 \mathrm{~A} \& 1 \mathrm{~F}$ ), level of hepatocellular ballooning (Figure $1 \mathrm{C} \& 1 \mathrm{H}$ ) and fibrosis (Fig 1E \& $1 \mathrm{~J}$ ). Consistent with its regulation by estrogens ${ }^{26}$, BMP8B is also expressed at higher levels in females; on the contrary, it appears reduced in patients with type 2 diabetes mellitus (T2D) under medical treatment (metformin with/without biguanides, incretins or TZDs); details in Figure 1 A-J. Full details of the population in Supplementary Table 2. As observed in rodents, human BMP8B protein is expressed in $\mathrm{K} 18^{+}$hepatocytes and aSMA ${ }^{+} \mathrm{HSC}$ and pericytes (Figure $1 \mathrm{~K}$ ). These results indicate that BMP8B is induced in HSC and hepatocytes in response to acute/chronic liver damage, raising the question if BMP8B signaling may contribute to liver disease progression.

\section{HSC and Primary Hepatocytes up-regulate Bmp8b in vitro}

Consistent with the in vivo observations, levels of $B m p 8 b$ mRNA expression in primary mouse hepatocytes $(\mathrm{PH})$ were low under basal conditions at baseline, and comparable to liver tissue (Extended Data Figure 2). However, murine PH strongly upregulated Bmp8b mRNA when 
cultured in vitro (Extended Data Figure 2A); preferentially when cultured at lower density, a condition known to favor $\mathrm{PH}$ proliferation and de-differentiation (Extended Data Figure 2B \& 3C) ${ }^{27}$. Interestingly, data from publicly available databases [GSE122660 28; Extended Data Figure 2D] suggested that fatty acids and/or pro-inflammatory factors did not further modulate Bmp8b levels either in human $\mathrm{PH}$ or in hepatocyte cell lines. In primary murine HSCs, BMP/TGF $\beta$ receptors and intracellular effectors (Smads) were abundant but relatively stable during trans-differentiation into activated myofibroblasts (Next Generation Sequencing, NGS; Extended Data Figure 3A and 3B; Supplementary Table 5). Ingenuity "Upstream Regulator" Analysis (URA; Extended Data Figure 3C) confirmed the promotion of Tgf $\beta$ signaling suggested by the transcriptional regulation of multiple BMP/TGF $\beta$ ligands during the HSC activation process (Extended Data Figure 3B); Bmp8b upregulation was transient and peaking at day 4; its upregulation was enhanced by palmitic acid but not by other modulators of HSC activation (Extended Data Figure 3D). Bmp8b mRNA was undetectable in Kupffer Cells and in circulating inflammatory cells.

\section{Bmp8b activates both branches of BMP/TGF $\beta$ signaling in primary HSC}

We then studied which hepatic cells sense BMP8B and its mechanism of action. Previous reports have shown that BMP8s act as pan BMP/TGF $\beta$ receptors agonists and activate Smad2/3 and Smad1/5/9 17,18,29, but this information has never been confirmed in the liver.

We first studied the capability of primary murine HSC (Figure 2) and AML12 (Supplementary Figure 2) to sense BMP8B: in line with previous evidences showing that BMP8s bind all Type 1 and Type 2 receptors ${ }^{18}$, BMP8 activated both Smad 2/3 and 1/5/9 in an ALK-dependent manner (Figure 2A-D) also promoting the expression of Smad targets (Figure 2E) in HSC. Conversely, BMP8 did not activate Smads in AML12 cells (Supplementary Figure 2 A-B). Furthermore, markers of proliferation, apoptosis, differentiation, epithelial/mesenchymal transition, and pro-inflammatory pathways were not affected in BMP8-stimulated primary hepatocytes (Supplementary Figure 3). These results suggested that hepatocyte-derived 
Vacca et al, Bmp8b in NASH

Bmp8b might not signal in an autocrine fashion on murine hepatocytes, but instead potentially modulate other cell types.

\section{Bmp8b is an autocrine and paracrine signal promoting HSC activation in vitro}

Given the expression profile of Bmp8b during HSC activation, we then investigated its involvement in this process. Using primary HSCs freshly isolated from Bmp8b KO and WT mice and cultured in vitro, we observed that the absence of Bmp8b delayed the timeassociated increase in aSMA mRNA (Figure 3A) and protein (Figure 3B \& 3C). Genetic ablation of Bmp8b also decreased the transcription of collagens, pro-inflammatory cytokines, and multiple downstream effectors of Smad signaling without changing Tgf $\beta 1$ expression (Figure 3A). The defective production of $M c p 1(C c / 2)$ and Rantes (Cc/5) is relevant in liver pathophysiology given that these chemokines drive the recruitment of inflammatory cells, promote wound-healing responses, and their downstream receptors are candidate targets for NASH treatment ${ }^{30}$. Ingenuity "Pathway Analysis" (IPA) and URA from fully activated HSCs (NGS; Day 8) confirmed that Bmp8b ablation reduced the activation of pro-inflammatory pathways and TGF $\beta$ signaling (Figure 3D; expanded TGF $\beta$ network in Figure 3E; NGS data analysis in Supplementary Table 6). Moreover, analysis of the media from cultured HSC (Figure 3F) revealed defective extracellular matrix production/processing (PRO-C3, Activated MMP9, and C3M) and release of inflammatory mediators (II6 and Mcp1).

While direct BMP8 treatment (alone or in combination with LPS) did not affect Murine Bone Marrow Derived Macrophages (BMDM; Supplementary Figure 4A \& 4B; suggesting that BMP8 does not activate the inflammasome directly, or indirectly via TLR4), conditioned media from HSCs induced in BMDM Smad 2/3 target genes (Socs3) and pro-inflammatory genes such as Mcp1 (Supplementary Figure 4C); this effect was less pronounced challenging BMDM with Bmp8b KO HSC conditioned media. These data suggest that Bmp8b, mediating the secretion of pro-inflammatory cytokines in HSC, might indirectly modulate inflammatory cells behavior, with repercussions on the wound healing responses to liver injury. 
Recombinant BMP8 rescued aSMA mRNA/protein levels, as well as the expression of genes involved in ECM remodeling (Extended Data Figure 4A-C), inflammation (Extended Data Figure 4D), and Smad signaling (Extended Data Figure 4E) in the Bmp8b KO HSC. Intriguingly, BMP8 accelerated some features of HSC activation in WT cells, suggesting that Bmp8b self-produced by HSCs is not saturating their intracellular signaling, and that Bmp8b produced by other cells may also be modulating HSC behavior.

Taken together, these results confirm that both endogenous Bmp8b production, and the ectopic administration of the recombinant protein (mimicking a paracrine effect), are able to promote HSC trans-differentiation into myofibroblasts. Furthermore, defective activation of KO HSCs leads to reduced ECM deposition and, most importantly, to impaired inflammatory pathways in HSC, further implicating Bmp8b in the pathogenesis of liver disease.

\section{Genetic ablation of Bmp8b tunes the wound healing response in the acute $\mathrm{CCl}_{4}$ model}

We thus investigated whether the absence of Bmp8b also modulated the wound healing response in vivo following acute $\mathrm{CCl}_{4}$ treatment. In WT mice, acute $\mathrm{CCl}_{4}$ injury induced $\mathrm{Bmp} 8 \mathrm{~b}$ mRNA in liver, peaking three days after the insult but remaining elevated at day 5 (Figure 4A). At day 3 after $\mathrm{CCl}_{4}, B m p 8 b$ was one of the most strongly upregulated TGF $\beta / B M P$ family ligands (Figure 4B; NGS). Bmp8b was produced by hepatocytes and HSCs (Figure 4C; FISH), in agreement with our in vitro and human observations. Bmp8b absence prevented the $\mathrm{CCl}_{4}$ driven increase in liver weight (LW/BW\%; Figure 4D) and tended to decrease ALT 3 days after $\mathrm{CCl}_{4}$ injection (Figure 4E). Despite showing similar levels of hepatocellular damage to WT littermates (Figure 4F \& S10A, in red), three days post $\mathrm{CCl}_{4}$ injection Bmp8b KO livers showed reduced inflammatory infiltrates (Figure $4 G$ \& Supplementary Figure $5 A$; in yellow; semiquantitative histologic score in Supplementary Table 3), and a defective infiltration of lymphocytes (CD3+) and myeloid (Ly6CG+) cells (IHC, Figure 4H \& Supplementary Figure 5B; immune cell deconvolution on NGS, Figure 4I). Moreover, at both time points (more predominantly at day 5), Bmp8b KO mice exhibited reduced aSMA positive myofibroblasts (Figure 4J \& Supplementary Figure 5C; aSMA IHC staining). Being this a model of wound 
healing response to acute hepatic injury, also featuring compensatory hepatocyte regeneration, and given there processes are heavily modulated by inflammatory pathways ${ }^{30}$ and BMP/TGF $\beta$ signaling ${ }^{11}$, we next investigated the effect of Bmp8b on hepatocyte proliferation: the absence of Bmp8b resulted in reduced hepatocytes proliferation (PCNA IHC; Figure $4 \mathrm{~K}$ and Supplementary Figure 5D) and de-differentiation (Afp; Figure 4L). Gene expression analysis (Figure $4 \mathrm{M} \& 4 \mathrm{~N}$ ) confirmed the anticipated effects of $\mathrm{CCl}_{4}$ in promoting inflammation, HSC activation, and compensatory regeneration in WT mice. However, all these changes were attenuated in Bmp8b KO mice. Taken together, these results confirmed that the absence of Bmp8b limited HSC activation, preventing immune cell infiltration; during the resolution phase of the wound healing response (Day 5) to acute injury, Bmp8b KO livers showed a faster recovery from hepatic damage and reduced tissue repair responses.

\section{Genetic ablation of Bmp8b delays liver regeneration after Partial Hepatectomy (PHx)}

We next investigated whether genetic ablation of Bmp8b delayed liver regeneration irrespectively of the challenge used to promote compensatory hepatocyte proliferation. We used the PHx model where the regeneration is primed predominantly by resident immune cells (compared to the acute $\mathrm{CCl}_{4}$ model that is characterized by a large component of inflammatory infiltrates). $\mathrm{PHx}$ induces a peak in hepatocyte proliferation 3 days after surgery; this process is dependent by inflammatory priming and modulated by TGF $\beta / B M P$ signals ${ }^{11,31}$. Bmp8b mRNA and protein expression was significantly increased during the regenerative phase and, consistent with other models, hepatocytes and pericytes/HSC were identified as the cellular source of Bmp8b; in this model, the protein staining appeared to be more intense in hepatocytes (Figure 5A-D). Bmp8b was the most significantly up-regulated BMP/TGF $\beta$ family member after PHx (Figure 5B), and its absence led to defective liver regrowth (LW/BW\%; Figure 5E). Hepatocyte proliferation, was reduced in Bmp8b KO mice (vs. WT; PCNA staining, Figure 5F \& 5H; mitotic figures, H\&E, Figure 5G \& 5I). Gene expression analysis (Figure 5J \& $5 \mathrm{~K}$ ) was consistent with reduced hepatocyte proliferation (reduced Ccnd1 and Ccne1 mRNA; increased p21 $\mathrm{mRNA}$ ) and impaired ECM remodeling (reduced $\mathrm{Mmp9}$ ). As with $\mathrm{CCl}_{4}$ 
treatment, Bmp8b KO livers showed defects in inflammatory [II6 and Stat3 are also crucial for the priming of liver regeneration ${ }^{31}$ ] and proliferation pathways (Figure $5 \mathrm{~K}$ ).

These results confirmed in the PHx model that the absence of Bmp8b ${ }^{11,32}$ is associated with defective hepatic inflammatory pathways and compensatory hepatocyte proliferation.

\section{Bmp8b promotes NASH in human 3D in vitro NASH micro-tissues}

We next investigated in vitro whether BMP8B contributed to NASH pathophysiology and progression. We used a novel in vitro microphysiological system (MPS) consisting of 3D perfused micro-tissues of primary human cells ( $\mathrm{PH}$, Kupffer cells - KC, and HSC) challenged with a mix of free fatty acids (FFA) to mimic $\mathrm{NASH}^{25,33}$. This in vitro model displayed high homology with in vivo NASH models (Figure $6 \mathrm{~A} ; ~ 80 \%$ of expressed genes overlapping with the WD model) and contained highly differentiated hepatocytes expressing high levels of albumin, apolipoproteins, clotting factors, and metabolic genes (Top 250 genes and differential analysis in Supplementary Table 7); AFP was expressed at low levels. The MPS expressed a repertoire of BMP/TGF $\beta$ effectors (Figure 6B) and ligands (Figure 6C) and developed lipid droplets when challenged with FFA (Figure 6D). Finally, BMP8B was expressed in this model; FFA challenge promoted a modest and not significant upregulation of BMP8B (Extended Data Figure $5 \mathrm{~A}$ ). To mechanistically study the contribution of BMP8B to $\mathrm{NASH}$, we administered recombinant BMP8 with/without ALKs inhibitors (TGF $\beta$ and BMP7 were used as positive controls) to the FFA-challenged MPS, and performed phospho-proteomics (Extended Data Figure 5B) and transcriptomics (5h incubation; NGS, Figure 6E and Supplementary Table 7; RTqPCR, Fig 6F). As previously observed in murine HSC, BMP8 induced the phosphorylation of SMAD1 and SMAD3 (Extended Data Figure 5B) and the transcription of their targets; these effects were prevented by ALK inhibitors (Figure 6F). Using IPA/URA (NGS; Figure 6E), we focused on biological mechanisms differentially regulated by BMP8 which were clustered into three groups. The first cluster ("BMP-like") and included pathways and upstream regulators promoted by BMP8 and BMP7 with modulation reduced/prevented by K02288; this cluster included pro-inflammatory pathways (IL1, NFkB, CCR3) and drivers of cell proliferation and/or 
survival (CSF2, Telomerase signaling, AMPK, mTOR). The "TGF $\beta$-like" cluster (modulated by BMP8 and TGF $\beta$; modulation reduced/prevented by A-8301) featured known TGF $\beta$ effectors (ERK1/2, SMAD2\&4, P38 MAPK, JUNB), growth factors (FGF, EGFR, NGF, HGF, VEGFA), and pro-inflammatory and pro-fibrotic pathways. Finally, the "BMP/TGF $\beta$-like" cluster (modulated by BMP8, BMP7 and TGF $\beta$; modulation reduced/prevented by both ALK inhibitors) featured inflammatory pathways (STAT4, FOS, INFG, JAK/STAT), growth factors signaling (CSF1/3, PDGF, GM-CSF), and proliferation/survival/carcinogenesis (JNK, AKT, FOS). The change of the phosphorylation status of some of these hits (Figure 6E, in bold) was also confirmed experimentally by targeted phospho-proteomics (Extended Data Figure 5B). These results indicate that BMP8 rapidly activated both TGF $\beta / B M P$ dependent signaling also promoting multiple aspects of the wound-healing response driving NASH progression ${ }^{30,34}$.

We next studied the effects of "long-term" (48h) stimulation by daily administration of BMP8, TGF $\beta$ or BMP7 (RTqPCR: Figure 6G and Extended Data Figure 5C; secretome: Figure 6H, 6I, Extended Data Figure 5D; NGS, Extended Data Figure 5E and Supplementary Table 7). We confirmed that BMP8 promotes the transcription (Figure 6G) of pro-inflammatory chemokines (RANTES and MCP1), and of drivers of cell proliferation (MYC, CCNE1) featuring a "BMP-like" behavior ${ }^{11}$. However, in this "chronic" setting, BMP8 showed a neutral effect on canonical markers of HSC activation (as shown by the lack of modulation of aSMA, TIMP1, and COL1A1; Figure 6G) and had a weak promoting effect on ID1 (while having a neutral effect, or even a suppressive effect, on Smad 2/3 targets; Extended Data Figure 5C). The analysis of the secretome in the culturing media (48h; Figure 6H and 6l; Extended Data Figure 5D) demonstrated that BMP8 promoted the release of ALT, M-CSF, RANTES and MCP1, and suppressed the release of anti-inflammatory cytokines IL-10 and IL-13, while only mildly (and not-significantly) inducing TIMP1 and C3M excretion. We next compared the effects of BMP8 at $5 \mathrm{~h}$ vs. $48 \mathrm{~h}$ by NGS (Extended Data Figure 5E). These analyses confirmed a consistent modulation at both time-points of pathways involved in inflammatory responses and cell cycle control. On the other hand, in agreement also with the BMP/TGF $\beta$ targets (Extended Data 
Figure 5C) and fibrotic genes (Figure 6G) at 48h, TGF $\beta$-like pathways and pro-fibrotic signals either ceased to be regulated at $48 \mathrm{~h}$ (ERK 1/2) or followed a biphasic response (activation at 5h, suppression at 48h: SMAD2, JUNB, PDGFB), thus suggesting that these pathways are only transiently modulated by BMP8.

Together these results confirmed, in an advanced MPS human 3D in vitro NASH model, that ectopic BMP8 treatment activated BMP/TGF $\beta$ pathways to promote wound healing responses; however, in these settings, only mild and transient effects on HSC activation and fibrosis processes were observed.

\section{Absence of Bmp8b attenuates NASH progression in vivo}

To determine if the aforementioned mechanisms were pathophysiologically relevant, we next studied the contribution of BMP8B to NASH progression in vivo in the WD model of NASH F1 fibrosis [a model of NASH fibrosis that recapitulates the hepatic lipid profile characteristic of $\mathrm{NASH}$ patients ${ }^{35}$. As observed in the acute injury models, Bmp8b was one of the most upregulated TGF $\beta$ /BMP ligands (Figure 7A-C: RTqPCR, NGS, and FISH), and produced in

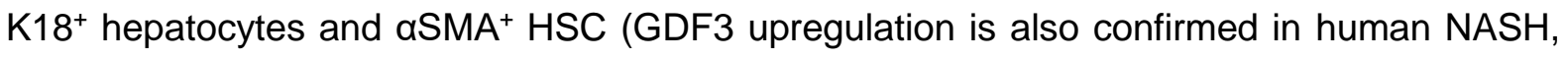
Supplementary Figure 6). We used male Bmp8b KO mice because, in contrast to KO female mice, they exhibit a similar energy balance compared to WT controls ${ }^{16,17}$ thus avoiding confounding effects of obesity and adipose tissue function on NASH progression. As previously described ${ }^{16,17}$, male Bmp8b KO mice were indistinguishable from WT littermates with respect to BW and body composition (Supplementary Figure 7A), metabolic biochemistry (Supplementary Figure 7B), adipose tissue weight (Supplementary Figure 7C), food intake (Supplementary Figure 7D), energy expenditure (Supplementary Figure 7E), liver weight (Figure 7D) and hepatic lipid composition (Supplementary Figure 7F) on both WD and Low Fat Control Diet (LFD). However, the livers of Bmp8b KO mice fed WD exhibited less NASH activity than their WT littermates, as indicated by 1) reduced ALT (Figure 7E); 2) decreased "activity" component of the SAF score with both hepatocyte ballooning and lobular inflammation scores reduced (Figure 7F \& 7G; details in Supplementary Figure 8A-B); and 3) 
diminished fibrosis (Figure $7 \mathrm{H} \&$ 7l); a trend toward reduced fibrosis was also observed in circulating PRO-C3 (Figure 7J), a non-invasive circulating biomarker of hepatic fibrosis in $\mathrm{NASH}^{36}$. In both genotypes, the pattern of fibrosis was peri-sinusoidal ${ }^{5}$. Further immunoprofiling of the inflammatory infiltrates in WD-fed mice (Figure $7 \mathrm{~K} \& 7 \mathrm{~L}$ ) and immune cell-type deconvolution analysis (NGS; Figure 7M) was indicative of a defective infiltration of B-cells (CD45R+), lymphocytes (CD3+), and myeloid cells (Ly6CG+), while KC were unchanged (F4/80+; Supplementary Figure 8C). Also in WD, Bmp8b KO mice had reduced expression of pro-inflammatory (Rantes) and pro-fibrotic (collagens/Timp1) genes in response to WD, while aSMA mRNA (Figure 7N) and protein (IHC; Supplementary Figure 8D) were unchanged. IPA (NGS; Figure 7O) confirmed that Bmp8b KO mice in WD exhibited defective upregulation of multiple pro-inflammatory/pro-fibrotic/proliferative pathways when compared to WT littermates. Taken together, these data show that absence of Bmp8b attenuated NASH in NASH with mild fibrosis.

To investigate the effect of the absence of Bmp8b in advanced NASH-fibrosis, we challenged Bmp8b KO and WT mice with a Choline Deficient High Fat Diet (CDHFD; Extended Data Figure 6), known to induce F2 fibrosis within $12+$ weeks $^{37}$. As previously observed, Bmp8b KO mice were comparable to WT mice with regards to BW (Extended Data Figure 6A), liver to BW ratio (Extended Data Figure 6B), and metabolic profile (Extended Data Figure 6C). In contrast to the WD model, we did not observe protection from liver damage in Bmp8b KO mice: liver histology and circulating ALT and PRO-C3 levels were similar between genotypes (Extended Data Figure 6D-J). However, gene expression profiling of the whole liver (Extended Data Figure 6K) and of freshly isolated HSC (Extended Data Figure 6L) confirmed our previous observations of an impaired activation of the TGF $\beta$ signaling, and a reduced expression of pro-inflammatory genes and markers of HSC activation. These results suggested that the absence of Bmp8b had an impact on the activation of the TGF $\beta / B M P$ signaling and inflammation in HSC, but either inhibiting Bmp8b might not be powerful enough to interfere 
with the fibrotic processes once they have been chronically activated, or that the protection from fibrosis might be context/model dependent.

\section{Comparative NGS analysis identifies wound healing responses as the key pathways modulated by Bmp8b in vivo}

Last, we characterized the Bmp8b mediated mechanisms common to acute $\mathrm{CCl}_{4}, \mathrm{PHx}$, and WD, three models with strong and diverse effects on the hepatic wound-healing responses, to identify model-independent functions of Bmp8b. Using IPA/URA (Figure 8A-C; Details in Supplementary Table 8), we focused on processes modulated by all the three challenges, and by the presence/absence of Bmp8b. Bmp8b KO mice exhibited impaired activation of TGF $\beta$ downstream effectors, pathways/upstream regulators controlling inflammation, liver regeneration/hepatocellular carcinogenesis, and fibrosis/HSC cell transactivation. Notably, the activation status of most of these pathways was mirrored by recombinant BMP8 in the MPS NASH model (Figure 6E).

Comparing the genes significantly modulated in these models (Figure 8D), we also retrieved a list of hits significantly modulated at least in two of the models (KO vs. WT comparison) and showing the same direction of modulation in the three groups; we identified 36 "shared" genes (Figure $8 \mathrm{E}$ ) featuring modulators of inflammation and/or NASH progression [e.g. Mcp1/ Ccl2 38,39, or OPG/Tnfrsf11 $b^{40,41}$ ], TGF $\beta$ signaling/HSC activation/fibrosis [e.g. Col4a1/2 ${ }^{42}$ or Mcp1/ Ccl2 ${ }^{38,39}$ ], proliferation/cancer [such as the VEGF target Angpt2 ${ }^{43}$, or $\mathrm{Ngfr}^{44}$ ]. Similarly, genetic ablation of Bmp8b prevented the suppression of genes usually expressed in healthy livers [FA elongation, sterol metabolism, bile acid transporters, and P450 coenzymes] including PPARa targets [Acot1 ${ }^{45}, C y p 2 c 8^{46}$, Sult2a ${ }^{47}$ ]. PPARs upregulation (also featured by IPA; Figure $8 \mathrm{~A}$ ) is particularly intriguing as PPARs exert anti-inflammatory and anti-fibrotic activities in $\mathrm{NASH}$, and are candidate targets for $\mathrm{NASH}$ treatment ${ }^{48}$.

Taken together these data confirm that preventing the de novo induction of Bmp8b (or inhibiting its function) might be sufficient to attenuate inflammation and chronic wound healing 
responses; BMP8B could thus serve as a candidate therapeutic strategy to delay NASH development and progression.

\section{DISCUSSION}

The incidence and prevalence of NASH is rising due to the twin epidemics of obesity and the Metabolic Syndrome. This emerging trend is occurring just as the other great global driver of chronic liver disease, viral hepatitis, is on the wane due to advances in HBV vaccination and HCV treatments. NASH usually occurs in the context of hepatic lipotoxicity leading to hepatocellular damage that in turn causes compensatory proliferation, a pro-inflammatory and pro-fibrotic microenvironment, ultimately leading to cirrhosis and HCC $3,49,50$.

We found that BMP8B was marginally expressed in the normal liver, but it was the member of the TGF $\beta / B M P$ pathways most-consistently upregulated among acute and chronic hepatic injury models; we also found that BMP8B upregulation was disease-stage-dependent in murine models (Supplementary Table 1) and also in patients (Figure 1) with NASH. Our results point to BMP8B being an important mediator of NASH progression, as it drives both branches of the SMAD signaling pathway, and it promotes inflammation, wound healing responses, and NASH progression. To untangle the effect of Bmp8b upregulation in these processes, we first studied Bmp8b KO HSCs and treated them with BMP8 (Figure 2 and 3; Extended Data Figure 4): we found that absence of Bmp8b was sufficient to attenuate HSC activation and their inflammatory phenotype and that recombinant BMP8 rescues their pro-inflammatory and profibrotic phenotype. HSCs contribute to liver inflammation, facilitating the recruitment of inflammatory cells, and producing multiple cytokines and chemokines ${ }^{30}$; intriguingly the absence of Bmp8b, also reduced the potential of the HSCs to modulate inflammatory cells behavior (Supplementary Figure 4C) ${ }^{30}$. Bmp8b thus was a likely contributor to the early events mediating HSC activation, triggering the pro-inflammatory and pro-fibrotic responses ${ }^{30}$ in both, liver injury and NASH. Using Bmp8b KO mice, we have shown that absence of Bmp8b leads to defective HSC activation, inflammation and compensatory regeneration in acute models of 
liver injury and/or hepatocyte loss $\left(\mathrm{CCl}_{4}\right.$ and $\left.\mathrm{PHx}\right)$ indicating that $\mathrm{Bmp} 8 \mathrm{~b}$ may be a crucial effector of wound healing responses orchestrating liver repair ${ }^{30}$.

We investigated whether upregulation of Bmp8b in NASH was pathophysiologically relevant for NASH progression. As HSC activation is a consequence of the transition from NAFL to $\mathrm{NASH}$, their contribution to the wound healing responses in NASH might have implicated Bmp8b in early phases of NASH progression ${ }^{48,49}$. We posited that the selective proinflammatory effect of Bmp8b, together with its capacity to promote HSC activation, could exert a central regulatory function determining NASH progression. In support of this hypothesis we demonstrated, in both human and murine pre-clinical NASH models, that Bmp8b modulation directs the TGF $\beta / B M P$ signaling pathways, towards promoting proinflammatory responses, cell proliferation (crucial for both liver regeneration and potentially hepatocellular carcinogenesis), and HSC activation (Figures 6-8; Extended Data Figure 6). The comparative NGS analysis (Figure 6 and 8) confirmed a comprehensive role of BMP8B modulating TGF $\beta / B M P$ downstream effectors ${ }^{11}$, pathways and upstream regulators involved in inflammation, proliferation/carcinogenesis, HSC activation and fibrosis ${ }^{30}$. Whereas the effect of Bmp8b is more evident in the early pro-inflammatory stages, its influence on relatively late events such as fibrosis may be less relevant as suggested by both, the in vitro MPS and the CDHFD models. This may probably be due to compensatory mechanisms occurring in advanced $\mathrm{NASH}$, and/or due to the extreme redundancy of TGFB/BMP signaling.

Given that Bmp8b is a secreted protein, not required for the normal function of the liver and selectively induced in liver disease, we propose that preventing the induction of BMP8B and/or blocking its extracellular activity could be a safe strategy to limit the progression of NASH and, potentially, of other liver diseases. More studies are needed to identify at which stage humans could benefit the most of this treatment and to develop an efficient in vivo inhibition that selectively targets BMP8B without interfering with the beneficial functions of the TGF $\beta / B M P$ system in liver (patho)-physiology. However, based on our results, we speculate that the 
inhibition of BMP8B could be beneficial to prevent the shift from NAFL to NASH and in the early phases of $\mathrm{NASH}$ progression to prevent HSC activation and inflammation.

LEAD CONTACT FOR MATERIAL AND CORRESPONDENCE: Further information and requests for resources and reagents should be directed and will be fulfilled by Dr Michele Vacca (mv400@medschl.cam.ac.uk).

ACKNOWLEDGMENTS: The authors are indebted to the following colleagues and institutions: A) MRC Metabolic Diseases Unit [MC_UU_00014/5]: Martin Dale, Mark Campbell, Rita Dias, the Disease Model Core (DMC; Hannah Webber, Daniel Hart, Sarah Grocott, Charley Beresford, Darren Jessop, Eerika Rasijeff \& Amy Warner), the Biochemistry Assay Lab (Keith Burling and collaborators), the Genomics and Transcriptomics Core (Marcella Ma), the Histology Core (James Warner) and the Imaging Core (Gregory Strachan). B) MRC Human Nutrition Research and Department of Biochemistry, University of Cambridge: Francis Sanders, Zoe Hall, and James West. C) Histopathology/ISH core facility of Cancer Research UK - Cambridge Institute: Julia Jones for assistance with in situ hybridization. The human NASH histological samples come from the Human Research Tissue Bank of the Cambridge University Hospitals, which is supported by the NIHR Cambridge Biomedical Research Centre. MV, JLG, AVP are supported by MRC programs (MRC MDU Programme Grant. PO 4050281695 "Lipotoxicity and the Metabolic Syndrome" and MRC DMC MC UU 12012/2 to AVP; Lipid Profiling and Signalling, MC UP A90 1006 to JLG) and MRC adjunct funding as part of the Cambridge Initiative in Metabolic Diseases (Lipid Dynamics and Regulation: MC_PC_13030). MV, MA and AVP are also supported by the Cambridge NIHR Biomedical Research Center (Gastroenterology); MV is recipient of the BRC Gastroenterology PumpPriming award 2018/2019 that founded part of this study. FO is supported by MRC program Grants MR/K0019494/1 and MR/R023026/1. JL is supported by Medical Research Council PhD studentship and a CRUK program grant (C18342/A23390). QMA, MV, AVP, VR, MA and DT are contributing members of the European NAFLD Registry. QMA is supported by the Newcastle NIHR Biomedical Research Centre (BRC). MV has been fellow of the Fondazione 
Umberto Veronesi in 2014. MA, AVP, and JLG received funding from the Evelyn Trust. MV, OG, DT, MA, FO, QMA, MJN DJL, and AVP are members of the EPoS (Elucidating Pathways of Steatohepatitis) consortium, which is funded by the Horizon 2020 Framework Program of the European Union under Grant Agreement 634413.

AUTHOR CONTRIBUTIONS: M.V. and A.V.P. conceived and designed the study and wrote the manuscript. M.V., S.V. and V.P. designed and performed the WD experiment. J.L., F.O., M.V., and S.V. designed and performed the PH \& $\mathrm{CCl}_{4}$ experiments. M.V., S.S., T.K., Z.T. and K.P. performed the in vitro experiments. O.G., Q.M.A., V.R., M.A., and S.D. contributed with human data and samples. D.T. and S.D. scored the liver histology. Z.T. and W.L. contributed to the design of some of the in vitro experiments. M.J.N. and D.J.L. performed the Collagen3/MMP9 assays. Z.A. and J.L.G. performed liver lipidomics. B.Y.H.L. and M.V. analyzed the NGS sequencing data. M.V. performed most of the analyses in all the models. All the authors provided useful criticism during the study, and critically reviewed the manuscript.

COMPETING INTERESTS STATEMENT: At the time of this study, TK and SS were employees of CN Bio Innovations Ltd; MJN and DJL of Nordic Bioscience and are among original inventors and patent holders for the PRO-C3, C3M and MMP9 assays. F.O is a director of Fibrofind limited. J.L and F.O are shareholders in Fibrofind limited. The other authors declare no competing interest.

\section{References}

1. Younossi, Z., et al. Global burden of NAFLD and NASH: trends, predictions, risk factors and prevention. Nat Rev Gastroenterol Hepatol 15, 11-20 (2018).

2. Azzu, V., Vacca, M., Virtue, S., Allison, M. \& Vidal-Puig, A. Adipose tissue-liver cross talk in the control of whole-body metabolism: implications in non-alcoholic fatty liver disease. Gastroenterology (2020).

3. Vacca, M., Allison, M., Griffin, J.L. \& Vidal-Puig, A. Fatty Acid and Glucose Sensors in Hepatic Lipid Metabolism: Implications in NAFLD. Semin Liver Dis 35, 250-261 (2015).

4. Bedossa, P., et al. Histopathological algorithm and scoring system for evaluation of liver lesions in morbidly obese patients. Hepatology 56, 1751-1759 (2012).

5. Kleiner, D.E., et al. Design and validation of a histological scoring system for nonalcoholic fatty liver disease. Hepatology 41, 1313-1321 (2005).

6. Andriopoulos, B., Jr., et al. BMP6 is a key endogenous regulator of hepcidin expression and iron metabolism. Nat Genet 41, $482-487$ (2009). 
7. Wang, W., Yang, Y., Meng, Y. \& Shi, Y. GDF-3 is an adipogenic cytokine under high fat dietary condition. Biochem Biophys Res Commun 321, 1024-1031 (2004).

8. Cunha, S.I., Magnusson, P.U., Dejana, E. \& Lampugnani, M.G. Deregulated TGFbeta/BMP Signaling in Vascular Malformations. Circ Res 121, 981-999 (2017).

9. David, L., Feige, J.J. \& Bailly, S. Emerging role of bone morphogenetic proteins in angiogenesis. Cytokine Growth Factor Rev 20, 203-212 (2009).

10. Bonnardel, J., et al. Stellate Cells, Hepatocytes, and Endothelial Cells Imprint the Kupffer Cell Identity on Monocytes Colonizing the Liver Macrophage Niche. Immunity (2019).

11. Herrera, B., Addante, A. \& Sanchez, A. BMP Signalling at the Crossroad of Liver Fibrosis and Regeneration. Int J Mol Sci 19(2017).

12. Hernanda, P.Y., et al. SMAD4 exerts a tumor-promoting role in hepatocellular carcinoma. Oncogene 34, 5055-5068 (2015).

13. Zheng, Y., et al. Bone morphogenetic protein 2 inhibits hepatocellular carcinoma growth and migration through downregulation of the PI3K/AKT pathway. Tumour Biol 35, 51895198 (2014).

14. Zhang, Y., Alexander, P.B. \& Wang, X.F. TGF-beta Family Signaling in the Control of Cell Proliferation and Survival. Cold Spring Harb Perspect Biol 9(2017).

15. Kreidl, E., Ozturk, D., Metzner, T., Berger, W. \& Grusch, M. Activins and follistatins: Emerging roles in liver physiology and cancer. World J Hepatol 1, 17-27 (2009).

16. Martins, L., et al. A Functional Link between AMPK and Orexin Mediates the Effect of BMP8B on Energy Balance. Cell Rep 16, 2231-2242 (2016).

17. Whittle, A.J., et al. BMP8B increases brown adipose tissue thermogenesis through both central and peripheral actions. Cell 149, 871-885 (2012).

18. Wu, F.J., et al. BMP8A sustains spermatogenesis by activating both SMAD1/5/8 and SMAD2/3 in spermatogonia. Sci Signal 10(2017).

19. Cheng, Z., et al. BMP8B mediates the survival of pancreatic cancer cells and regulates the progression of pancreatic cancer. Oncol Rep 32, 1861-1866 (2014).

20. Mima, K., et al. Gene expression of bone morphogenic protein 8B in the primary site, peripheral blood and bone marrow of patients with gastric cancer. Oncol Lett 6, 387-392 (2013).

21. Saito, T., et al. Genetic variations in humans associated with differences in the course of hepatitis C. Biochem Biophys Res Commun 317, 335-341 (2004).

22. Tryndyak, V., et al. Interstrain differences in the severity of liver injury induced by a cholineand folate-deficient diet in mice are associated with dysregulation of genes involved in lipid metabolism. FASEB J 26, 4592-4602 (2012).

23. Stoyanov, E., et al. Chronic liver inflammation modifies DNA methylation at the precancerous stage of murine hepatocarcinogenesis. Oncotarget 6, 11047-11060 (2015).

24. Cubero, F.J., et al. TNFR1 determines progression of chronic liver injury in the IKKgamma/Nemo genetic model. Cell Death Differ 20, 1580-1592 (2013).

25. Kostrzewski, T., et al. Three-dimensional perfused human in vitro model of non-alcoholic fatty liver disease. World J Gastroenterol 23, 204-215 (2017).

26. Grefhorst, A., et al. Estrogens increase expression of bone morphogenetic protein $8 \mathrm{~b}$ in brown adipose tissue of mice. Biol Sex Differ 6, 7 (2015).

27. Nakamura, T., Yoshimoto, K., Nakayama, Y., Tomita, Y. \& Ichihara, A. Reciprocal modulation of growth and differentiated functions of mature rat hepatocytes in primary culture by cell--cell contact and cell membranes. Proc Natl Acad Sci U S A 80, 7229-7233 (1983).

28. Breher-Esch, S., Sahini, N., Trincone, A., Wallstab, C. \& Borlak, J. Genomics of lipid-laden human hepatocyte cultures enables drug target screening for the treatment of nonalcoholic fatty liver disease. BMC Med Genomics 11, 111 (2018).

29. Pellegrinelli, V., et al. Adipocyte-secreted BMP8b mediates adrenergic-induced remodeling of the neuro-vascular network in adipose tissue. Nat Commun 9, 4974 (2018).

30. Pellicoro, A., Ramachandran, P., Iredale, J.P. \& Fallowfield, J.A. Liver fibrosis and repair: immune regulation of wound healing in a solid organ. Nat Rev Immunol 14, 181-194 (2014). 
31. Fausto, N., Campbell, J.S. \& Riehle, K.J. Liver regeneration. Hepatology 43, S45-53 (2006).

32. Kan, N.G., Junghans, D. \& Izpisua Belmonte, J.C. Compensatory growth mechanisms regulated by BMP and FGF signaling mediate liver regeneration in zebrafish after partial hepatectomy. FASEB J 23, 3516-3525 (2009).

33. Kostrzewski, T., et al. A Microphysiological System for Studying Nonalcoholic Steatohepatitis. Hepatol Commun 4, 77-91 (2020).

34. Schuster, S., Cabrera, D., Arrese, M. \& Feldstein, A.E. Triggering and resolution of inflammation in NASH. Nat Rev Gastroenterol Hepatol 15, 349-364 (2018).

35. Hall, Z., et al. Lipid zonation and phospholipid remodeling in nonalcoholic fatty liver disease. Hepatology 65, 1165-1180 (2017).

36. Boyle, M., et al. Performance of the PRO-C3 collagen neo-epitope biomarker in nonalcoholic fatty liver disease. JHEP Rep 1, 188-198 (2019).

37. Matsumoto, M., et al. An improved mouse model that rapidly develops fibrosis in nonalcoholic steatohepatitis. Int J Exp Pathol 94, 93-103 (2013).

38. Baeck, C., et al. Pharmacological inhibition of the chemokine CCL2 (MCP-1) diminishes liver macrophage infiltration and steatohepatitis in chronic hepatic injury. Gut 61, 416-426 (2012).

39. Krenkel, O., et al. Therapeutic inhibition of inflammatory monocyte recruitment reduces steatohepatitis and liver fibrosis. Hepatology 67, 1270-1283 (2018).

40. Bosselut, N., et al. Including osteoprotegerin and collagen IV in a score-based blood test for liver fibrosis increases diagnostic accuracy. Clin Chim Acta 415, 63-68 (2013).

41. Yilmaz, Y., et al. Serum levels of osteoprotegerin in the spectrum of nonalcoholic fatty liver disease. Scand J Clin Lab Invest 70, 541-546 (2010).

42. Mann, J. \& Mann, D.A. Transcriptional regulation of hepatic stellate cells. Adv Drug Deliv Rev 61, 497-512 (2009).

43. Faillaci, F., et al. Liver Angiopoietin-2 Is a Key Predictor of De Novo or Recurrent Hepatocellular Cancer After Hepatitis C Virus Direct-Acting Antivirals. Hepatology 68, 1010-1024 (2018).

44. Scheving, L.A., Zhang, X., Stevenson, M.C., Threadgill, D.W. \& Russell, W.E. Loss of hepatocyte EGFR has no effect alone but exacerbates carbon tetrachloride-induced liver injury and impairs regeneration in hepatocyte Met-deficient mice. Am J Physiol Gastrointest Liver Physiol 308, G364-377 (2015).

45. Franklin, M.P., Sathyanarayan, A. \& Mashek, D.G. Acyl-CoA Thioesterase 1 (ACOT1) Regulates PPARalpha to Couple Fatty Acid Flux With Oxidative Capacity During Fasting. Diabetes 66, 2112-2123 (2017).

46. Thomas, M., et al. Peroxisome proliferator-activated receptor alpha, PPARalpha, directly regulates transcription of cytochrome P450 CYP2C8. Front Pharmaco/6, 261 (2015).

47. Feng, L., et al. Identification and characterization of a novel PPARalpha-regulated and 7alpha-hydroxyl bile acid-preferring cytosolic sulfotransferase mL-STL (Sult2a8). J Lipid Res 58, 1114-1131 (2017).

48. Hardy, T., Anstee, Q.M. \& Day, C.P. Nonalcoholic fatty liver disease: new treatments. Curr Opin Gastroenterol 31, 175-183 (2015).

49. Brunt, E.M., et al. Nonalcoholic fatty liver disease. Nat Rev Dis Primers 1, 15080 (2015).

50. Anstee, Q.M., Reeves, H.L., Kotsiliti, E., Govaere, O. \& Heikenwalder, M. From NASH to HCC: current concepts and future challenges. Nat Rev Gastroenterol Hepatol 16, 411-428 (2019). 
Vacca et al, Bmp8b in NASH

\section{FIGURE LEGENDS}

Figure 1: BMP8B is overexpressed in Human NASH according to disease stage and is expressed in both hepatocytes and stellate cells (HSC). Relative mRNA expression levels of $B M P 8 B$ measured by quantitative real-time polymerase chain reaction (A-E; RTqPCR; FJ: Nanostring) in the RNA extracted from liver biopsies of two independent cohorts of NASH patients. The levels of $B M P 8 B$ transcript increase with disease progression according to the NASH activity score (NAS; $\mathbf{A} \& \mathbf{F})$. The components of the NAS score show that the main drivers of $B M P 8 B$ expression are hepatocellular ballooning $(\mathbf{C} \& \mathbf{H})$, fibrosis stage $(\mathbf{E} \& \mathbf{J})$, gender, and the diagnosis/treatment of T2D. K) Representative IF of BMP8B, K18 (mainly hepatocytes) and aSMA (activated HSC/pericytes) protein expression in FFPE liver biopsies of $\mathrm{NASH}$ subjects at different stages of the disease $(\mathrm{N}: 6 ; 2-3$ needle biopsies specimens/patient stained; Magnification: 20x). BMP8B is expressed in NASH fibrosis, and co-localizes with both K18 and aSMA (White arrows indicate co-localization with aSMA). Results are shown as mean \pm standard error ( $\mathrm{n}$ : 40 Cambridge Cohort; $\mathrm{n}$ : 113 Newcastle/Paris cohort); expression data of biological replicates represented as dot plots. Statistical significance $(p<0.05)$ was assessed by Multivariate Analysis of Variance (MANOVA) with histological variables, gender and T2D as covariates. Full details of the population in Supplementary Table 2.

Figure 2. BMP8B activates both Smad $2 / 3$ and Smad $1 / 5 / 9$ signaling pathways in Bmp8b KO HSC. Primary murine Bmp8b KO HSC (confluence 35,000 cells $/ \mathrm{cm}^{2}$ ) activated in vitro (Day 7) were treated, after 3h FBS starvation, with/without recombinant human BMP8 protein 30-75 ng/mL, and/or ALK 1/2/3/6 inhibitor (K02288, $1 \mu \mathrm{M}$ ) or ALK 4/5/7 inhibitor (A$8301,5 \mu \mathrm{M})$ to study Smad $2(\mathbf{A}, \mathbf{C})$ or Smad 1/5/9 (B, D) phosphorylation after 30 min incubation. TGF- $\beta(5 \mathrm{ng} / \mathrm{mL})$ or BMP7 $(5 \mathrm{ng} / \mathrm{mL})$ were used as positive controls. Immunofluorescence (Magnification: 20x) was used to study phosphorylated Smads (in green); aSMA (in red) was used to stain the cytoplasm, Hoechst 33342 (blue) to stain the nucleus. The \% of positive nuclei was assessed by Harmony High Content Imaging and 
Analysis Software (PerkinElmer). Gene expression (E; Relative mRNA expression levels of TGF $\beta /$ BMP targets measured by RTqPCR) was assessed after $5 \mathrm{~h}$ of incubation with ligands and inhibitors. All the results are shown as mean \pm standard error; expression data of biological replicates are represented as dot plots. The one-way analysis of variance (ANOVA) plus Fisher's least significant difference multiple-comparison test was used to estimate the statistical significance among treatments (4 biological replicates/group; each biological replicate is a pool of three livers). Lowercase letters indicate post-hoc analysis significance: "a" means reference group; when groups show different letters, they should be considered statistically different at the post-hoc comparison; groups showing the same letter are statistically not-significant at the post-hoc comparison.

Figure 3. Genetic ablation of Bmp8b attenuates HSC transactivation in vitro. Freshly isolated HSC from Bmp8b KO mice and WT littermate mice were cultured for 4, 6 and 8 days at a density of $35 \mathrm{~K}$ cells $/ \mathrm{cm}^{2}$. Gene expression (A; RTqPCR) and aSMA protein expression (B\&C; IF; Magnification: 10X) were studied to investigate HSC's activation status (4 biological replicates/group; each biological replicate is a pool of three livers). Prediction of "Canonical Pathways" and "Upstream Regulators" (D) significantly enriched and predicted as activated (red) or inhibited (blue) according to IPA/URA in fully activated (Day 8) HSC (Details the NGS analysis in Supplementary Table 6); E) graphical representation in a network format of the changes of TGF $\beta$ targets levels suppressed in the NGS dataset in KO (vs. WT) cells. (F) Profiling of the culturing media (WT: 3 biological replicates/group; KO: 4 biological replicates/group; each biological replicate is a pool of cells from three murine livers): collagen 3 secretion and processing [proposed as marker of NASH-Fibrosis ${ }^{36}$ ] was assessed studying PRO-C3, MMP9 and C3M assays; secretion of pro-inflammatory mediators was assessed quantifying II6 and Mcp1 (Rantes was below detectability) in the culturing media at multiple time points (Day 4, 8, 11 of culturing). Relative mRNA expression of genes measured by RTqPCR or NGS; protein quantification was performed by immunofluorescence and quantified using ImageJ 1.8.0. Sequencing data are shown in a heatmap format representing 
the "activation" (Z) score [inhibition (blue) or activation (red)] of pathways/upstream regulators predicted by IPA/URA. All the other results are shown as mean \pm standard error; biological replicates are represented by dots. Statistical significance was assessed by Multivariate Analysis of Variance (MANOVA) using time-point and genotype as covariates.

Figure 4. Inflammation, stellate cells activation and hepatocyte proliferation are attenuated in $\mathrm{Bmp8b} \mathrm{KO}$ livers injured with $\mathrm{CCl}_{4}$. Bmp8b $\mathrm{KO}$ and littermate mice received a single olive oil (OO) or $\mathrm{CCl}_{4} \mathrm{IP}$ injection, and were culled 3 and 5 days later [n/group (refers to all the comparisons): (OO-WT) 6; (CCL4-WT3D) 4; (CCL4-KO3D) 6; (CCL4-WT5D) 7; (CCL4-KO5D) 5]. A) Relative Bmp8b mRNA expression measured by RTqPCR (whole liver). B) Relative TGF $\beta / B M P$ family ligands mRNA expression (3 days post injection) measured by NGS (the heatmap represents relative basal expression level - blue<yellow<orange -, the dotted graph represents Log2FC; Details in Supplementary Table 8). C) In situ hybridization (2 replicates; staining repeated twice) reveals that, upon $\mathrm{CCl}_{4}$ challenge, $\mathrm{Bmp} 8 \mathrm{~b}$ mRNA is expressed by K18+ cells (mainly hepatocytes) and aSma+ cells (mainly activated HSC and pericytes) - Magnification: 40X. D) Liver to body weight ratio (LW/BW\%) is increased by $\mathrm{CCl}_{4}$; Bmp8b KO mice show less pronounced liver enlargement. $\mathrm{CCl}_{4}$-treated $\mathrm{Bmp} 8 \mathrm{~b} \mathrm{KO}$ mice show a trend in reduced serum alanine aminotransferase (ALT; E) levels three days after the challenge. HALO imaging software analysis on the whole-tissue $(\mathrm{H} \& \mathrm{E})$ suggests that $\mathrm{CCl}_{4}$ induces a substantial increase of hepatocellular damage (F; HALO quantification) and inflammation (G; HALO quantification) peaking 3 days after the injection; Bmp8b KO mice showed reduced inflammation at the same time point. Further IHC immune cell profiling $(\mathbf{H}$; Day 3) suggests a reduced infiltration in the Bmp8b KO livers from CD3+ (mostly lymphocytes) and of Ly6CG+ (mostly myeloid) cells in the KO liver. This result is also confirmed by the "immune cell-type deconvolution analysis" performed on the NGS data (I). We also profiled by IHC a Smooth Muscle Actin staining (aSMA; J) and Proliferating Cell Nuclear Antigen IHC staining (PCNA; K) that appeared reduced in Bmp8b KO mice compared to WT littermates, especially at Day 5. Furthermore, in light with decreased 
compensatory proliferation, also circulating levels of $\operatorname{Afp}(\mathbf{L})$ appeared reduced in the Bmp8b KO mice; M \& N) Relative mRNA expression (whole liver RNA) levels of genes measured by RTqPCR and IPA on the NGS data (Day 3) confirm reduced inflammation, stellate cells activation and proliferation in Bmp8b KO mice. Representative images of $\mathrm{H} \& \mathrm{E}$ and IHC in Supplementary Figure 5. Supplementary Table 4 shows that the lipid composition of the livers did not differ between WT and KO mice. All the results are shown as mean \pm standard error; biological replicates are represented as dot plots. Statistical significance was assessed either by one-way analysis of variance (ANOVA) plus Fisher's least significant difference multiplecomparison test, or by two-sided Student T-Test (and FDR correction for NGS data). Lowercase letters indicate post-hoc analysis significance: "a" means reference group; when groups show different letters, they should be considered statistically different at the post-hoc comparison; groups showing the same letter are statistically not-significant at the post-hoc comparison.

\section{Figure 5. Absence of Bmp8b leads to defective liver regeneration in the Partial} Hepatectomy (PHx) model. Bmp8b KO and littermate mice received $\mathrm{PHx}$ and were culled 3 days later [n/group (for all the comparisons): DO-WT: 5; DO-KO: 4; D3-WT: 5; D3-KO: 4]. A) Relative Bmp8b mRNA expression measured by RTqPCR. B) Relative TGF $\beta$ family ligands mRNA expression ( 3 days post injection) measured by NGS (the heatmap represents relative basal expression level - blue<yellow<orange -, the dotted graph represents Log2FC; Details in Supplementary Table 8). C-D) FISH (Magnification: 40X; 2 replicates; staining repeated twice) and IF (2 replicates; staining repeated twice) reveal that after $\mathrm{PHx}, \mathrm{Bmp8b}$ mRNA and protein are expressed by K18+ cells (mainly hepatocytes) and aSMA+ cells (mainly activated HSC and pericytes). E) Liver to body weight ratio (LW/BW \%) shows that Bmp8b KO mice have reduced regenerative potential compared to WT littermates (resected liver in red as reference). Defective hepatocyte proliferation was also confirmed by reduced Proliferating Cell Nuclear Antigen IHC staining (PCNA quantified by HALO imaging software analysis on the whole-tissue; staining repeated once; Magnification: 10X; F \& H) and reduced 
number of mitotic figures (H\&E staining repeated once; Magnification: 20X; G \& I). Relative mRNA expression levels (whole liver RNA; resected livers from the same mice were used as D0 reference) of genes measured by $\operatorname{RTqPCR}(\mathbf{J})$ and IPA pathway analysis (K; NGS) confirm reduced inflammation and compensatory proliferation in Bmp8b $\mathrm{KO}$ mice during the proliferative phase of liver regeneration. All the results are shown as mean \pm standard error; replicates are represented as dot plots. Statistical significance was assessed either by oneway analysis of variance (ANOVA) plus Fisher's least significant difference multiplecomparison test, or by two-sided Student T-Test (and FDR correction for NGS data). Lowercase letters indicate post-hoc analysis significance: "a" means reference group; when groups show different letters, they should be considered statistically different at the post-hoc comparison; groups showing the same letter are statistically not-significant at the post-hoc comparison.

Figure 6. BMP8 stimulates TGF $\beta / B M P$ signaling in a human 3D in vitro NASH model promoting inflammation and proliferation. Human primary liver cells $(\mathrm{PH}, \mathrm{KC}$, and $\mathrm{HSC})$ were cultured in an in vitro NASH model (LiverChip ${ }^{\mathrm{TM}}$ - CN Bio Innovations) consisting of 3D perfused micro-tissues of primary human cells challenged with a medium containing a mixture of FFAs, in the presence of physiologically relevant quantities of insulin and sugars to induce a "NASH-like" phenotype. (A) Comparison of the transcriptomic data (NGS) of this in vitro model with the in vivo WD model of NASH (in Figures 7\&8) shows high homology ( $80 \%$ overlapping genes); details on differentially expressed genes in Supplementary Table 7: this model expresses a plethora of TGF $\beta / B M P$ effectors (B) and ligands (C), comparable to in vivo NASH models, and develops lipid droplets as a consequence of FFA challenge as observed through Oil Red O staining (example picture; Magnification: 10X; staining repeated multiple times giving similar results) (D). One week after fat challenge, cells were treated with/without recombinant Human BMP8 protein $(75 \mathrm{ng} / \mathrm{mL})$, with/without ALK 1/2/3/6 inhibitor (K02288, $1 \mu \mathrm{M})$ or ALK 4/5/7 inhibitor (A-8301, $5 \mu \mathrm{M})$ to study TGFB/BMP-related gene expression changes at $5 \mathrm{~h}$ (E: NGS data). F: RTqPCR confirmation of TGF $\beta / B M P$ targets 
(long-term consequences) at 48h (G: RTqPCR; NGS in Extended Data Figure 5E). Treatment with TGF $\beta(5 \mathrm{ng} / \mathrm{mL})$ or BMP7 $(5 \mathrm{ng} / \mathrm{mL})$ were used as positive controls. All the results are shown as mean \pm standard error (biological replicates are represented as dot plots) or in a heatmap matrix representing the "activation" Z-score of "Canonical Pathways" and "Upstream Regulators" significantly $(\mathrm{p}<0.05)$ enriched and predicted as activated (red) or inhibited (blue) according to IPA. To provide a framework of interpretation, the data have been clustered in "BMP-like" (modulated -significantly enriched and with a -2<Z-score $>2$ - by both BMP8 and BMP7; modulation reduced/prevented by K02288), "TGF $\beta$-like" (modulated by both BMP8 and TGF $\beta$; modulation reduced/prevented by A-8301) and "BMP/TGF $\beta$-like" (modulated by BMP8, BMP7 and TGF $\beta$; modulation reduced/prevented by both ALK inhibitors). Two days after culturing with challenges, we also studied the cell secretome in the culturing media $(\mathbf{H}$, I, Inflammatory factors and markers of collagen deposition and remodeling; additional data in Extended Data Figure 5D; PRO-C3 was undetectable). Statistical significance (4 biological replicates/group) was assessed by two-sided T-test (E; each treatment vs control) for the NGS data, or by one-way analysis of variance (ANOVA) plus Fisher's least significant difference multiple-comparison test $(F-I)$. Lowercase letters indicate post-hoc analysis significance: "a" means reference group; when groups show different letters, they should be considered statistically different at the post-hoc comparison; groups showing the same letter are statistically not-significant at the post-hoc comparison.

Figure 7. Attenuated inflammation and fibrosis in Bmp8b KO mice challenged with WD to model NASH/F1. Bmp8b KO and wild-type littermate mice were treated for 32 weeks with WD (9WT \& 8KO) or Low-Fat Diet (LFD; 8WT \& 6KO) control. Relative mRNA expression levels of Bmp8b and other TGF $\beta$ ligands was measured by RTqPCR (A) or NGS (B; the heatmap represents relative basal expression level - blue<yellow<orange -; the dotted graph represents Log2FC; detailed NGS analysis in Supplementary Table 8) in murine livers. The levels of Bmp8b mRNA transcript are almost absent in the normal liver and highly expressed in WD-induced NASH in K18+ cells (mainly hepatocytes) and aSma+ cells (mainly activated 
HSC and pericytes) by $F I S H(C ; 2$ replicates; staining repeated twice; magnification: 40X) as also observed at protein level (Extended Data Figure 1). D) Liver to body weight \% ratio (LW/BW\%) is increased by WD with no genotype-associated effects. WD-treated Bmp8b KO mice show reduced serum alanine aminotransferase (E; ALT), SAF activity score (F\&G; details in Supplementary Figure 8A \& 8B), and Fibrosis (H\&I; PSR stain quantified using "Indica Lab" HALO imaging software on the whole-tissue scanned slide). (J) A trend in reduced circulating PRO-C3, a non-invasive biomarker of fibrosis, is also observed in WDchallenged Bmp8b KO mice. (K \& L) IHC immune cell profiling (WD) suggests a reduced infiltration in the KO livers from CD3+ (mostly lymphocytes), Ly6CG+ (mostly myeloid) cells, and CD45R+ (mostly B) cells in WD-challenged KO livers; this result is also confirmed by the "immune cell-type deconvolution analysis" (M) performed on the NGS data. Relative mRNA expression levels (whole liver RNA) of genes involved measured by RTqPCR (N) and IPA analysis (O; NGS) confirm reduced inflammation, fibrosis and stellate cells activation in WDchallenged Bmp8b KO mice. All the results are shown as mean \pm standard error; biological replicates are represented as dot plots. Statistical significance was assessed by either twotailed Student T-Test (plus FDR for NGS), one-way analysis of variance (ANOVA) plus Fisher's least significant difference multiple-comparison test, or by Kruskal-Wallis one-way analysis of variance on ranks plus Dunn's multiple-comparison test $(\mathbf{G})$, when relevant. Lowercase letters indicate post-hoc analysis significance: “a” means reference group; when groups show different letters, they should be considered statistically different at the post-hoc comparison; groups showing the same letter are statistically not-significant at the post-hoc comparison.

Figure 8. NGS analysis confirms on a large scale that absence of Bmp8b impacts proinflammatory, and pro-fibrotic, and proliferative pathways in $\mathrm{CCl}_{4}, \mathrm{PHx}$ and WD models. Gene expression profiled by NGS, analyzed with two-sided T-test (sample size as defined in figures $4,5,7$ ), and analyzed by IPA in Bmp8b KO mice and WT mice following $\mathrm{CCl}_{4}$ (3 days), $\mathrm{PHx}$ (3 days), and WD (32 weeks) challenges. Details in Supplementary Table 
8. Prediction of "Canonical Pathways" (A) and "Upstream Regulators" (B) significantly enriched and predicted as activated (red) or inhibited (blue) according to IPA/URA. Data are shown as a heatmap matrix format representing the "activation" (Z) score prediction by IPA. C) Graphical representation in networks of differentially modulated genes (green: downregulated; red: up-regulated in the WD - KO vs. WT comparison) leading to the predicted ($2<$ Z-score $>2$ ) inhibition (blue) of the "upstream regulators" according to IPA. D) Comparing the genes differentially modulated in the treated Bmp8b KO mice (vs. WT), a subset of 171 genes was modulated in at least 2 of the three datasets - i.e. differentially modulated $(p<0.05$; $-0.378<\log 2 \mathrm{FC}>0.378$ ); of these genes, 36 hits $(\mathrm{E})$ show the same direction of regulation in all the three datasets, so being most-directly associated to Bmp8b absence independently from the challenge. Data are shown in a heat-map with a matrix format representing the regulation (Log2FC KO vs WT; yellow: up; green: down). 


\section{METHODS}

\section{Study population (Patients with NASH)}

The "Cambridge" Cohort consisted of 40 consecutive male patients recruited at the NASH Service at the Cambridge University Hospital. The "Newcastle/Paris" Cohort consisted of 113 patients recruited at the Freeman Hospital, Newcastle upon Tyne Hospitals NHS Foundation Trust under ethical approval EPoS-UK (REC 15/NE/0150) and Hospital Pitié-Salpêtrière, Sorbonne Université. All the patients had a clinical diagnosis of NAFLD (patients with alternate diagnoses and etiologies were excluded), histology scored by either DT or SD according to the NASH CRN Scoring System [NAS ${ }^{5}$, and snap-frozen tissue for research purposes. This study was approved by the relevant Ethics Committees in the participating institutions. All patients gave their informed consent for the use of data (Biochemistry and clinical history) and samples for research purposes; the principles of the Declaration of Helsinki were followed. Description of the study population is given in Supplementary Table 2.

\section{Animals}

All data are from male mice. C57BI6/J mice were purchased from Charles River. Bmp8b KO mice were generated as previously described ${ }^{51}$ on a C57BI6/J background and compared to wild-type littermates. Mice were maintained in a temperature-controlled room $\left(21^{\circ} \mathrm{C}\right)$ with a 12 $\mathrm{hr}$ light/dark cycle with free access to diet and water in pathogen-free facilities compliant with the FELASA Guidelines ${ }^{52}$. The UK Home Office and the USC Bioethics Committee of the Universities of Cambridge and Newcastle approved all animal procedures. Mice were fed on a chow diet (Safe Diets, Code ds-105) until they were enrolled into specific procedures.

\section{Western Diet Studies}

Western (Teklad, TD88137) and Low Fat Control (Teklad, TD08485) diets were administered ad libitum to 9 week old animals for a total duration of the challenge of 32 weeks. Fat and lean mass was calculated by time-domain nuclear magnetic resonance (TD-NMR) by using a 
minispec Live Mice Analyzer LF50 (Bruker) the day of the culling. Serum and tissue collection were performed in fed condition the day of the culling.

\section{Choline Deficient High Fat Diet Studies}

Bmp8b KO mice and Wild-Type littermates underwent 14 weeks feeding with L-Amino Acid Rodent Diet with $60 \mathrm{kcal} \%$ Fat and Modified Levels of Choline and Methionine formulated by Research Diets, Inc. (Product code: A06071302) to model NASH-Fibrosis ${ }^{37}$. Serum and tissue collection were performed in fed condition the day of the culling. The lateral left lobe of the liver was used for whole tissue analyses, the rest of the liver was used for HSC isolation. HSC GE analysis was studied in freshly isolated cells (i.e. not cultured).

\section{High Fat Diet Studies}

Five-week-old wild type mice were purchased from Harlan Laboratory Inc. (UK). Temperature was maintained at $21^{\circ} \mathrm{C}$ with a $12 \mathrm{hr}$ light/dark cycle. Seven lean controls were fed on regular chow diet (Rat \& Mouse No. I Maintenance; Special Diet Services, UK; diet code 801151) and seven in 55\% High Fat Diet (energy composition: $55 \%$ fat, $29 \%$ protein, and $16 \%$ carbohydrate; diet code: 829197; Special Diet Services, UK) for 14 days.

\section{Carbon Tetrachloride Studies}

Sixteen weeks old Bmp8b KO or WT underwent IP injection of $2 \mathrm{ul} / \mathrm{g}$ body weight of the $\mathrm{CCl}_{4}$ : olive oil $(1: 1(v / v))$ mix. Mice were humanely culled under isofluorane terminal anesthesia, 3 or 5 days after the IP injection, by cutting the heart and IVC. Blood was collected from the chest cavity. These experiments were approved by the Animal Welfare and Ethical Review Board and carried out at Newcastle University Comparative Biology Centre under a UK Home office license.

\section{Partial Hepatectomy (PHx)}

Twelve week-old mice underwent 2/3 partial hepatectomy following the method of Higgins and Anderson. The left lateral and median lobes were completely excised, and mice were 
Vacca et al, Bmp8b in NASH

humanely killed 3 days after the surgery during the expected peak of proliferation. Resected livers were used as time 0 control for gene expression analyses.

\section{Isolation and culture of primary hepatocytes}

Hepatocytes were isolated using a two-step collagenase perfusion method: after cervical dislocation of mice, liver were exposed by midline incision and perfused at $37^{\circ} \mathrm{C}$ in situ by cannulation of cava vein with a 26 G needle (Gilson 500 -flux flow peristaltic pump at 6.4 $\mathrm{mL} / \mathrm{min}$ ) with GIBCO Liver Perfusion Medium (Cat. No.: 17701, Invitrogen) containing 2\% P/S (Cat. No.15140) for approximately 10 min and, then GIBCO Liver Digestion Medium (Cat. No.: 17703, Invitrogen) for additional 8-10 minutes. The digested liver was then kept in Leibovitz's L-15 medium (Cat. No.: L1518, Sigma) containing 5\% FBS in ice until the hepatocytes were detached and filtered using a plastic mesh with 100 um pores. Hepatocytes were the centrifuged at 1500rpm for 2 minutes at $4^{\circ} \mathrm{C}$, washed twice with GIBCO hepatocyte wash medium (HWM) (Cat. No.: 17704, Invitrogen) containing 1\% PS, and then Percoll 42\% (Cat. No.: P1644, Sigma-Aldrich) in sterile PBS was used to remove dead cells. Live cells were then washed once with GIBCO hepatocyte wash medium (HWM) containing $1 \% \mathrm{PS}$, and once with hepatocyte attachment medium (HAM): Williams medium E (WME) in the presence of $5 \%$ FBS, 1\%PS, 1\% glutamine (pH 7.4). Cells were then seeded in BD Biocoat Collagen I 12-well Multiwell Plates (BD Biosciences, Cat 356500 ) at a density or 20,000 or 100,000 cells $/ \mathrm{cm}^{2}$ in HAM for additional $2 \mathrm{~h}$, and then maintained with the hepatocytes maintenance medium: Williams medium E (WME) in the presence of $5 \%$ FBS, $1 \%$ PS, $1 \%$ glutamine plus Primary Hepatocyte Maintenance Supplements (4\% ITS \& 70 ul of dexamethasone - Cat. No. CM 4000, Invitrogen) \pm BMP8 (30 ng/mL) recombinant protein (R\&D Cat 1073-BP).

\section{Isolation, culture and activation of primary Hepatocyte Stellate cells (HSC)}

Primary mouse HSCs were isolated from livers of Bmp8b KO or WT littermate controls. Liver tissue was digested with Pronase and Collagenase B (Roche) and the cell suspension was subsequently separated by an $11.5 \%$ Optiprep gradient (Sigma). HSCs were cultured into 
plastic (Corning) using Dulbecco's modified Eagle's medium supplemented with pyruvate (1\%), glutamine (1\%), penicillin/streptomycin (1\%) and heat-inactivated fetal bovine serum (during the activation process: 16\%; in fully activated HSCs: $10 \%$ ) - All reagents from Life Technologies. Freshly isolated (day 0) cells were considered quiescent and "day 7+" cultures regarded as fully activated. Cells were maintained in an incubator at $37^{\circ} \mathrm{C}$ with $5 \% \mathrm{CO}_{2}$. For the time-course experiments, HSC were grown at a confluence of 35,000 cells $/ \mathrm{cm}^{2}$ either on Corning well 6 plates (RNA) or (for IF) in Nunc® Lab-Tek® Permanox® plastic Chamber Slide $^{\mathrm{TM}}$ system (Sigma, C7182-1PAK) for the time-course during the activation process. The growing medium (with/without hBMP8 protein $30 \mathrm{ng} / \mathrm{mL}-\mathrm{R} \& \mathrm{D}$ Cat 1073-BP, or OA $100 \mu \mathrm{M}$, or PA $100 \mu \mathrm{M}$, or TNFa $30 \mathrm{ng} / \mathrm{mL}$, or LPS $50 \mathrm{ng} / \mathrm{ml}$, or PDGF $10 \mathrm{ng} / \mathrm{mL}$ ) was refreshed the day after the isolation, and every 2-3 days since then. Experiments involving fully activated HSCs were performed after HSC activation (Day 8) in T75 plastic Flasks (Corning); subconfluent $(70 \%)$ cells were then plated at a confluence of 35,000 cells $/ \mathrm{cm}^{2}$ either on Corning well 6 plates (RNA) or Perkin Elmer 96 Well Cell-carrier Ultra Plate (pSmads); after ON culture, and $3 \mathrm{~h}$ FBS starvation, the cells where treated (for $0.5 \mathrm{~h}$ for Smads phosphorylation; $5 \mathrm{~h}$ for RNA) in FBS-free culturing medium with/without recombinant Human BMP8 protein 30-75 $\mathrm{ng} / \mathrm{mL}$ (R\&D Cat 1073-BP), and/or ALK 1/2/3/6 inhibitor (K02288, $1 \mu \mathrm{M})$, and/or ALK 4/5/7 inhibitor (A-8301, $5 \mu \mathrm{M})$, or TGF- $\beta$ (5 ng/mL), or BMP7 (5 ng/mL).

\section{AML12 culturing, maintenance and treatment}

AML12 cells (ATCC® CRL-2254 ${ }^{\mathrm{TM}}$ ) were maintained in DMEM/F12 high glucose (Invitrogen, Cat: $11330-057$ ) supplemented with $10 \%$ FBS, $1 \%$ penicillin/streptomycin antibiotics, $1 \%$ ITS (Insulin-Transferrin-Selenium Supplement; Life technologies, Cat: 41400-045), 40 ng/ml dexamethasone (Sigma, Cat: D4902-25MG). For the experiment, the cells were plated at a density of 75,000 cells $/ \mathrm{cm}^{2}$ in growth medium on Corning well 6 plates (RNA) or Perkin Elmer 96 Well Cellcarrier Ultra Plate (pSmads). After 24h of FBS and dexamethasone starvation, cells were treated for $30 \mathrm{~min}$ (pSmads) or $5 \mathrm{~h}$ (RNA) with: Human BMP8 protein $30-75 \mathrm{ng} / \mathrm{mL}$ 
(R\&D Cat 1073-BP), and/or ALK 1/2/3/6 inhibitor (K02288, $1 \mu \mathrm{M})$, and/or ALK 4/5/7 inhibitor (A-8301, $5 \mu \mathrm{M})$, or TGF- $\beta$ (5 ng/mL), or BMP7 (5 ng/mL).

\section{Microphysiological System - 3D Human in vitro NASH model}

Cell cultures were performed in the microphysiological system (MPS), LiverChip ${ }^{\mathrm{TM}}$ (CN Bio Innovations, Welwyn Garden City, UK), as previously described ${ }^{25,33}$. Briefly, 3D microtissue cultures were performed with cryopreserved primary human cells [hepatocytes $(\mathrm{PHH}), \mathrm{Kupffer}$ cells (KC), and stellate cells (HSC)], purchased from Life Technologies (Paisley, UK). Cells were cultured for one week in HEP-FAT medium (CN Bio Innovations) which contains a mixture of saturated and unsaturated FFAs, as well as physiologically relevant quantities of insulin and sugars. Complete media changes were performed on all wells every $48 \mathrm{~h}$. After 8 days, microtissues were cultured for up to 48 hours in the presence of either: BMP8 (75

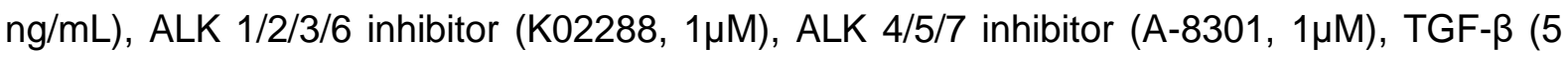
$\mathrm{ng} / \mathrm{mL})$ or BMP7 (5 ng/mL).

\section{Isolation, culture, differentiation, and treatment of primary bone-marrow derived macrophages}

Femur and tibia bones from WT mice were isolated and cleaned, and $10 \mathrm{~mL}$ of Roswell Park Memorial Institute Medium (RPMI)-1640 (Sigma) was flushed through each bone using a syringe. Bone marrow cells were counted manually, pelleted by centrifugation, and re suspended in RPMI-1640 with 20-30\% L929 conditioned medium, 10\% heat-inactivated FBS (Gibco, Thermo Fisher Scientific) and 100 units/mL penicillin/streptomycin (Thermo Fisher Scientific) (macrophage differentiation medium). To differentiate into macrophages, cells were seeded in $10 \mathrm{~cm}$ non-culture treated plates (Falcon) at a density of $5 \times 10^{6}$ cells per plate per $10 \mathrm{ml}$ of macrophage differentiation medium and cultured for 7 days at $37^{\circ} \mathrm{C}$ in $5 \% \mathrm{CO}_{2}$. On day 5 of differentiation, medium was removed and $10 \mathrm{ml}$ of fresh macrophage differentiation medium was added to each plate. On day 7 of differentiation, macrophages were detached using ice-cold PBS containing $1 \mathrm{mM}$ EDTA, counted manually, centrifuged at $500 \mathrm{~g}$, room 
temperature for $5 \mathrm{~min}$ and re-suspended in macrophage differentiation medium at the concentration of $5 \times 10^{5}$ cells $/ \mathrm{ml}$. Immediately after, cells were plated for experiments at the following densities: $100 \mathrm{ul}$ of cell suspension per well of 96 -well plate, $500 \mathrm{ul} /$ well of 24 -well plate, $1 \mathrm{~mL} /$ well of 12 -well plate, $2 \mathrm{~mL} /$ well of 6 -well plate and $10 \mathrm{~mL}$ per $10 \mathrm{~cm}$ plate.

To make L929 conditioned medium, L929 cells (CCL-1, ATCC) were seeded in DMEM supplemented with $10 \%$ heat-inactivated FBS, 100 units/mL penicillin-streptomycin and $2 \mathrm{mM}$ L-glutamine (Sigma) at a density of 500,000 cells per $50 \mathrm{ml}$ of medium per T175 tissue culture flask. Medium was harvested after 1 week of culture, and then $50 \mathrm{~mL}$ of fresh DMEM supplemented with $10 \%$ heat-inactivated FBS, 100 units/mL penicillin-streptomycin and $2 \mathrm{mM}$ L-glutamine was added onto cells and harvested 1 week later. Batches obtained after the first and second weeks of culture were mixed at a $1: 1$ ratio and stored at $-20^{\circ} \mathrm{C}$. Treatments were performed in FBS-free medium, with either human BMP8 protein at $75 \mathrm{ng} / \mathrm{mL}$ (R\&D Cat 1073BP); Duration of the treatment: (RNA) 1, 4, 8, 24h; (IL1 $\beta$ secretion) 24h; (HSC conditional medium (25\%) $24 \mathrm{~h}$.

\section{Energy Expenditure (EE)}

EE was measured by indirect calorimetry in an Oxymax calorimetry chamber (Columbus, Ohio) with 2.7 liter capacity, as previously described ${ }^{53}$. Briefly: the Oxymax chamber was housed within a larger temperature controlled cabinet. Room air to the chamber was $21^{\circ} \mathrm{C}$, continuously monitored, and fixed. Oxygen consumption and carbon dioxide production were measured using a custom built oxygen and carbon dioxide monitoring system. Measurements of oxygen concentration and carbon dioxide concentration in room air and air leaving each cage were measured every 11 minutes. Baseline gas exchange was recorded once steady state was achieved (at least 3 consecutive stable measurements). Mice were kept into the calorimetry chamber for $48 \mathrm{~h}$ and water/food consumption were also measured. Energy expenditure was then calculated using indirect calorimetry with the Elia and Livesey constants for respiratory quotient ${ }^{54}$. 


\section{Analyses in Serum and Culturing Media (biochemistry and pro-inflammatory panels)}

Metabolic biochemistry and transaminases were measured on the Dimension RXL analyzer (Siemens Healthcare) or Perkin Elmer DELFIA using reagents and calibrators purchased from Siemens. Free fatty acids were measured using the Roche Free Fatty Acid Kit (Code: 11383175001) modified to run in MicroTitre plate format. Insulin was measured using electrochemical luminescence immunoassay on the MesoScale Discovery immunoassay platform. Inflammatory markers were studied by Meso-Scale Discovery (MSD) multiplex assay kits. Assays were run in technical duplicates. A minimum of two quality-control samples were run in each assay. All these measurements were performed by the Biochemistry Assay Lab (CBAL) of the Metabolic Research Laboratories, University of Cambridge. PRO-C3, C3M and MMP9 were analyzed by Nordic Bioscience A/S (Herlev, Denmark) using competitive ELISAs for the measurement of formation of type III collagen in human and rodents (PRO-C3 and rPRO-C3), MMP-9 mediated fragment of type III collagen (C3M) and circulating active MMP9 (MMP9) as previously described for PRO-C3 and C3M ${ }^{55,56}$. The MMP-9 assay was run on a 96-well streptavidin plate coated with the appropriate biotinylated synthetic peptide dissolved in an optimized buffer and incubated for $30 \mathrm{~min}$ at $20^{*} \mathrm{C}$. Twenty microliter of peptide calibrator or sample was added to appropriate wells, followed by $100 \mu \mathrm{L}$ of conjugated monoclonal antibody raised against the N-terminal part of activated MMP9. The plate was incubated for $20+/-1 \mathrm{hr}$ at 2-8C. Finally, $100 \mu \mathrm{L}$ tetramethylbenzinidine (TMB) Sens (Kem-En-Tec cat. No. 4850) was added and the plate was incubated for $15 \mathrm{~min}$ at 20C. All incubation steps were done with gentle shaking at $300 \mathrm{rpm}$. After each incubation step, the plate was washed five times in washing buffer (20 mM Tris, $50 \mathrm{mM} \mathrm{NaCl}, \mathrm{pH}$ 7.2). The TMB Sens reaction was stopped by adding $100 \mu \mathrm{L}$ of $1 \% \mathrm{H}_{2} \mathrm{SO}_{4}$ and extinction was measured at $450 \mathrm{~nm}$ subtracting the background at $650 \mathrm{~nm}$. A calibration curve was calculated using a 4-paramtric fit model. All samples were measured within the detection range and with acceptable percent of coefficient of variation (CV \%). 


\section{Tissue Collection and Histology}

All animal tissues for protein or RNA extraction were frozen at time of collection unless otherwise stated. Samples for histology were placed in $10 \%$ buffered formalin overnight before transfer to $70 \%$ ethanol and later embedding in paraffin. Serial 4 um sections were obtained from FFPE blocks and extra-coated with paraffin to preserve tissue integrity.

\section{Histology}

After overnight at $37^{\circ} \mathrm{C}$, sections were dewaxed with xylene and $100 \%$ ethanol, and washed in running water for minimum 2 minutes. The sections were stained with standard hematoxylin \& eosin $(\mathrm{H} \& \mathrm{E})$ or Sirius Red histochemical stains. For Sirius Red: Picro-Sirius Red (Pioneer Research Chemicals, PRC/R/109) was applied for 1 hour; sections were rinsed in $1 \%$ acetic acid (in $\mathrm{dH}_{2} \mathrm{O}$ ) to "waterproof" red staining and remove excess. Fast Green $1 \%$ in $\mathrm{dH}_{2} \mathrm{O}$ (Sigma, F7252-25G) was then incubated for 15 seconds and excess stain was rinsed in acetic acid $1 \%$ in $\mathrm{dH}_{2} \mathrm{O}$ (Fisher Scientific, A/0360/PB17) to remove excess stain. Sections were then dehydrated in graded alcohols, cleared in Xylene, and mounted.

Oil Red O staining of 3D cultures from the MPS in vitro model was completed as previously described ${ }^{33}$. Briefly, scaffolds containing microtissues were fixed in 4\% PFA for 15 minutes, washed twice with $70 \%$ isopropanol and then stained for $1 \mathrm{~h}$ in Oil Red O solution (Sigma Aldrich, UK). Tissues were washed three times in $\mathrm{dH} 2 \mathrm{O}$ and twice in $70 \%$ isopropanol before Color bright field images of stained scaffolds were taken using an inverted light microscope (Leica, UK).

\section{Immunohistochemistry}

After overnight at $37^{\circ} \mathrm{C}$, sections were dewaxed with xylene and industrial methylated spirits, washed in running water for minimum 5 minutes, and kept always hydrated using TBST. The sections then underwent the following steps: 1) (Optional) Target/antigen retrieval 25min @ $97.5^{\circ} \mathrm{C}(\mathrm{pH} 6.0$, Vector $\mathrm{H}-3300$ or $\mathrm{pH} 9.0$, Vector H-3301) 2) Washes in TBST; 3) blocking endogenous peroxidase for 5 min (DAKO Real Peroxidase Blocking solution, Cat S2023); 4) 
washes in TBST; 5) Blocking serum for 20-30 minutes (Animal-Free Protein Block, Vector SP5030); 5) Primary antibody incubation for 60 minutes at RT or ON at $4^{\star} \mathrm{C}$ (PCNA, DAKO Cat: M0879 - diluted 1:100; aSMA, SIGMA Cat: A2547 - diluted 1: 100; CD3 [CD3-12], Abcam, Cat: ab11089 - diluted 1:800; CD45R [RA3-6B2], Abcam, Cat: ab64100 - diluted 1:1000; Ly6C/G, Abcam, Cat: ab2557 - diluted 1:100 in antibody diluent DAKO, S3022; F4/80 [Cl:A31] Rt a Mse mAb, Bio-Rad, Cat: MCA497, diluted 1:20); 6) washes in TBST for 5 min; 7) Incubation for 30 min with MOM ImmPress Polymer Reagent (VECTOR, Cat: MP-2400) or ImmPRESS HRP Polymer (Vector, MP-7451); 8) washes in TBST; 9) DAB solution (5-10 minutes) prepared following the manufacturer's instruction (VECTOR - PEROXIDASE SUBSTRATE KIT DAB, Cat: SK-5100; or, ImmPACT DAB, Vector SK-4105); 9) Washes in TBST; 10) incubation (1min) with DAKO REAL Hematoxylin (CAT: S2020). The sections where then washed in tap water, dehydrated in graded alcohols, cleared in Xylene, and mounted.

\section{Immunofluorescence}

$\mathrm{HSC} / \mathrm{PH}$ were washed in cold sterile PBS two times and fixated in $4^{\circ} \mathrm{C}$ paraformaldehyde $4 \%$ for 10 (cells) -20 (tissues) min. All tissues were FFPE embedded. After overnight at $37^{\circ} \mathrm{C}$, tissue sections were dewaxed with xylene and $100 \%$ industrial methylated spirits, washed in running water for minimum 5 minutes, and kept always hydrated using TBST; antigen revealing step (Citrate, $\mathrm{pH} 6$ ) at $97 \%$ for 20 mins was also performed in FFPE tissues. After PBS washing, cells/slides were treated with PBS-glycine $0.1 \mathrm{M}$ for $15 \mathrm{~min}$, membranes were permeabilized with TBS + Triton X-100 0.1\% pH 7.4. After blocking in Animal-Free Blocking solution (Vector SP-5030; 30 min at RT for HSC; 2h at RT for livers), tissues/cells were then incubated overnight $\left(-4^{\circ} \mathrm{C}\right.$, in agitation) with primary antibody diluted in in blocking buffer

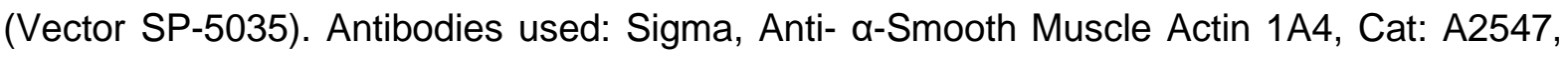
1:200; R\&D, Human/Mouse BMP-8b Antibody, Cat: AF6305, Concentration: $10 \mathrm{ug} / \mathrm{mL}$; Abcam, Anti-Cytokeratin 18, Cat: ab181597, Conc: 1:100; Abcam, Anti-Cytokeratin 18 [C-04], Cat: ab668,Conc: 1:100; Abcam, Anti-Mouse Cytokeratin 19 antibody [EPNCIR127B], Cat: 
ab133496, Conc: 1:100; R\&D, Anti-Human/Mouse Albumin Antibody, Cat:AF3329-SP, Conc: 10 ug/mL; Abcam, Anti-CD68 antibody, Cat: ab125212, Conc: 1:100; Thermo, VE-cadherin Polyclonal Antibody, Cat:36-1900, Conc: 5ug/mL; Cell Signaling, Phospho-SMAD2 (Ser465/Ser467) (E8F3R), Cat: 18338T, Conc: 1:300; Cell Signaling, Phospho-Smad1 (Ser463/465)/ Smad5 (Ser463/465)/ Smad9 (Ser465/467) (D5B10), Cat: 13820S, Conc: 1:100. After washing in TBST, and blocking as described in the previous sections, the secondary antibodies were incubated for $1 \mathrm{~h}$ at RT in blocking buffer, followed by Hoechst 33342 (Thermo, Cat: H3570; 1:2000 in TBS) for 5 minutes, and multiple TBST washes. Secondary antibodies used: Sigma, Anti-Rabbit highly cross-adsorbed CF ${ }^{\mathrm{TM}}$ 633, Cat: SAB4600132, Conc: 1:500; Sigma, Anti-Mouse highly cross-adsorbed CF ${ }^{\mathrm{TM}}$ 568, Cat: SAB4600075, Conc: 1:500; Thermo, anti-Sheep Cross-Adsorbed Alexa Fluor® 488, Cat: A11015, Con: 1:1000; Thermo, anti-Rabbit Highly Cross-Adsorbed, Alexa Fluor® 488, Cat: A21206. For the FFPE tissues, True View Quenching Kit (Vector, SP8400) was also applied for 3 minutes to reduce auto-fluorescence (murine studies). The slides where then mounted and kept at $4^{\circ} \mathrm{C}$. Pictures were taken with the Zeiss LSM 510 Meta (cells) confocal microscope, PerkinElmer Opera Phenix High Content Screening System (pSmads), or Zeiss AxioScan Z1 (tissues) at $10-40 x$, and analysis - percentage (\%) of marked area or percentage (\%) of marked nuclei - was performed using the ImageJ 1.8.0 software ${ }^{57}$, HALO (tissue slides) or using PerkinElmer Opera Phenix software (pSmads).

\section{Fluorescence in Situ Hybridization (FISH)}

Detection of mouse Bmp8 was performed on FFPE sections using Advanced Cell Diagnostics (ACD) RNAscope $\AA^{\circledR} 2.5$ LS Multiplex Reagent Kit (Cat No. 322800), RNAscope® 2.5 LS Probe- Mm-Bmp8b (Cat No. 570088) (ACD, Hayward, CA, USA). Briefly, sections were cut at $4 \mu \mathrm{M}$ thick, baked for 1 hour at $60^{*} \mathrm{C}$ before loading onto a Bond $\mathrm{RX}$ instrument (Leica Biosystems). Slides were deparaffinized and rehydrated on board prior to pre-treatments using Epitope Retrieval Solution 2 (Cat No. AR9640, Leica Biosystems) at $95^{\circ} \mathrm{C}$ for 15 minutes, and ACD Enzyme from the Multiplex Reagent kit at $40^{*} \mathrm{C}$ for 15 minutes. Probe 
Vacca et al, Bmp8b in NASH

hybridization and signal amplification was performed according to manufacturer's instructions. TSA plus-Fluorescein detection at 1:1000 (Akoya Biosciences Cat No. NEL741001KT) dilution of Bmp8b were performed on the Bond Rx according to the ACD protocol. Slides were then removed from the Bond $R x$ and placed into TBS in preparation for standard immunofluorescence counterstaining (K18 and aSma) that was performed as described in the previous sections.

\section{Tissue Imaging, Quantification, Scoring}

All the tissue slides were scanned using a Zeiss AxioScan Z1 and analyzed using HALO software (Indica Labs, Corrales, NM). The "Cytoplasmic \& Nuclear IHC quantification" module was used to quantify Nuclear PCNA (\% of total nuclei stained with DAB), CD45R, CD3, and LY6CG; and nuclear roundness/area thresholds were used to avoid false positives (e.g. PCNA staining of the nucleus of the inflammatory cells) when needed. aSMA and PSR staining (\% stained area) were quantified using the "Area Quantification" module. The "tissue Classifier module", utilizing a state-of-the-art machine learning algorithm to identify tissue types based on color, texture, and contextual features, was used to distinguish damaged liver (red), inflammatory infiltrates (yellow), healthy liver (green), and vessels (blue). The analyses were performed in the whole scanned section to avoid selection bias; tissue edges and vessels were excluded using appropriate tissue annotation and optical density settings in all the quantifications. Halo was "trained-by-example" on randomly selected images, and then the analysis was extended on the whole batch of sections with HALO's fully automatized and unbiased pipeline. WD, CDHFD, and $\mathrm{CCl}_{4}$ tissue slides were also scored by an expert liver pathologist (DT) in blind, using NASH CRN and SAF scoring systems for NAFLD/NASH or a necro-inflammatory score (range $0-6$ ) for $\mathrm{CCl}_{4}$-induced liver injury, as previously described $4,5,58$. 


\section{Enzyme-linked immunosorbent assay (ELISA) for IL-1 $\beta$}

BMDMs were treated as described in legend (Supplementary Figure 4) and culture supernatants were collected. IL-1 $\beta$ ELISA (DY401, R\&D Systems) was performed on the culture supernatants according to manufacturer's instructions.

\section{Targeted Phospho-Proteomics}

Protein phosphorylation in Extended Data Figure 5B has been evaluated on the lysates of the 3D co-cultures in Figure 6 and Extended Data Figure 5 treated for 30 min with BMP8; analysis was performed either with ELISA kits [SMAD1 (pS463/S465 + Total); Abcam, Cat: ab186035 \& Total Human Smad3 ELISA Kit; Novus Biologicals, Cat. NBP2-76622], or by Luminex xMAP Custom Phospho-Panel multiplexed assays (Cat: PR-CU060-KIT-17, Protavio Ltd, Cambridge); 3 biological replicates per treatment; 2 (ELISA) or 3 (Luminex) technical replicates per analysis. pSMADs were normalized against their total proteins; all the other proteins have been normalized against the average of total SMAD1 and SMAD3.

\section{RNA extraction and RNA integrity}

RNA form cells was isolated using miRNAeasy Mini Kits (Qiagen), according to the manufacturer's instructions. Tissue RNA (animal studies and human "Cambridge" cohort biopsies) was isolated using STAT-60 (AMS biotechnology, CS-502) according to the following procedure: tissue were homogenized in $1 \mathrm{ml}$ of STAT-60 using a tissue homogenizer, mixed by vortexing and centrifuged at $13,000 \mathrm{~g}(\mathrm{RT})$ for 5 minutes. The supernatant was then mixed by vortexing with chloroform (200 ul; Sigma, Cat 650471) and centrifuged at 12,000 g for 15 minutes $\left(4^{\circ} \mathrm{C}\right)$. The supernatant was then mixed with isopropanol (500 ul; Sigma, cat 33539 ) and centrifuged at $10,000 \mathrm{~g}$ for 10 minutes at $4^{\circ} \mathrm{C}$ to pellet RNA. The pellet was washed with $75 \%$ ethanol $(1 \mathrm{ml})$ and allowed to dry until ethanol had completely evaporated, and resuspended in RNAse free water (Thermo Fisher scientific, Delaware USA). Human "Newcastle/Paris" cohort biopsies were lysed using Trizol (Sigma-Aldrich, St. Louis, Missouri, USA) and mRNA was extracted with the Allprep DNA/RNA Micro kit (Qiagen, Venlo, The 
Netherlands). Samples were stored in aliquot at $-80^{\circ} \mathrm{C}$ prior to use. All reagents, plastic ware, and supplies used were sterile, nuclease free, and of molecular biology grade. RNA purity (A260/A280>1.80) and concentration were determined using Nanodrop spectrophotometer (Thermo Fisher scientific, Delaware USA). RNA integrity was studied using the 2100 Bioanalyzer System (Agilent) and RNA 6000 Nano or Pico Kits (Agilent, Santa Clara, California, USA). A RNA Integrity Number (RIN) of 7 or 8 was considered the lowest cut-off for RTqPCR and NGS, respectively.

\section{Lipid Extraction and Liquid Chromatography Mass Spectrometry for the Analysis of Intact Lipids}

Tissue samples were extracted using approximately $50 \mathrm{mg}$ of liver using an adaptation of the Folch methanol:chloroform extraction method ${ }^{59}$. Briefly: A metal bead (Qiagen Ltd., Manchester, UK) and $600 \mu \mathrm{L}$ of chloroform:methanol 2:1 solution was added to each sample tube. Samples were homogenized using a Tissue Lyser (Qiagen Ltd., Manchester, UK) for 10 minutes at $30 \mathrm{~Hz}$. Water $(400 \mu \mathrm{L})$ was added, after which the samples were homogenized for a further two minutes. Samples were sonicated in a water bath for 10 minutes. Finally, samples were centrifuged (3000 g, 10 minutes) and the organic and aqueous layers were carefully separated and dried under nitrogen.

A $10 \mu \mathrm{L}$ aliquot of the lipid extracts described above were diluted with $100 \mu \mathrm{L}$ of $2: 1: 1(\mathrm{v} / \mathrm{v} / \mathrm{v})$ of HPLC-grade isopropanol/acetonitrile/water. The instrumentation comprised of a Thermo Scientific Accela Autosampler coupled to a Thermo Scientific Elite ${ }^{\mathrm{TM}}$ Iontrap-Orbitrap Hybrid mass spectrometer with a heated electrospray ionization (HESI) source (Thermo Scientific, Hemel Hempstead Hertfordshire, UK). Separation of the triglyceride lipid species was achieved by injecting $5 \mu \mathrm{L}$ of each sample onto a Waters Acquity $\mathrm{BEH}(\mathrm{C} 18,50 \times 2.1 \mathrm{~mm}, 1.7$ $\mu \mathrm{m}$ ) column (Waters Ltd., Elstree, Hertfordshire, UK) maintained at $55^{\circ} \mathrm{C}$. Mobile phase A consisted of $10 \mathrm{mM}$ ammonium formate in acetonitrile: water (6:4), whilst mobile phase B contained $10 \mathrm{mM}$ ammonium formate in isopropanol: acetonitrile (9:1). The concentration of mobile phase B was increased from $40-99 \%$ in 4 min then equilibrated at $40 \%$ for 1 min with 
a flow rate of $1.0 \mathrm{~mL} / \mathrm{min}$. The HESI source was operated in positive ion mode with the source temperature maintained at $375^{\circ} \mathrm{C}$, the desolvation gas temperature was $380{ }^{\circ} \mathrm{C}$ and the gas flow rate was set at 40 arbitrary units. Mass spectrometric data was collected in the range 100-2000 m/z. Data processing was performed using the XCMS V 3.3 package (Bioconductor) within R (V 2.15.2, RStudio Inc, The R Foundation for Statistical Computing) to perform peak detection and alignment of the ions. The raw data was converted to the. $\mathrm{mzML}$ format using MSConvert V3 (ProteoWizard, http://proteowizard.sourceforge.net/news.shtml) and the script performed peak picking in the RT domain using the area under curve at fullwidth-half-maximum (FWHM) using an inbuilt library for peak assignment and annotation. The data was normalized to the relevant internal standards for all annotated peaks.

\section{Reverse transcription (RT-) polymerase chain reaction (PCR)}

$1000 \mathrm{ng}$ (tissues) or $250-500 \mathrm{ng}$ (cells) of RNA in $10 \mathrm{ul}$ of RNAse free water were used to generate cDNA according to manufacturer's protocol (Reverse Transcriptase System, Promega). All tubes are heated to $65^{\circ} \mathrm{C}$ for 5 minutes and returned to ice. Ten ul of RT mix were then added, composed as follows: 4 ul of M-MLV RT buffer (Promega M351A); 2 ul of 25mM MgCl 2 (Promega A351B); 2.5 ul nucleotide triphosphate (dNTP, Promega U151B); 0.5 ul $(100 \mathrm{mg} / \mathrm{ml})$ of random hexamers (Promega C118A); $1.0 \mathrm{ul}$ of reverse transcriptase (Promega M170b). Samples were incubated at $37^{\circ} \mathrm{C}$ for 1 hour. RT negatives were also used, as well as a titration curve from a pool of cDNAs (2 ul from each sample). Each sample was diluted 1:10 or 1:40 with RNAse free water. RT PCR was performed using TaqMan or SYBR green (Thermo) in a $13 \mathrm{ul}$ real time PCR reaction. For all experiments the following PCR conditions were used: denaturation at $95^{\circ} \mathrm{C}$ for $10 \mathrm{~min}$, followed by 40 cycles at $95^{\circ} \mathrm{C}$ for 15 seconds, then at $60^{\circ} \mathrm{C}$ for 60 seconds. All primers were designed on Primer Express v3.0 and available in Supplementary Table 9. Primers available on request. Reactions were run in duplicate for each sample and quantified in the ABI QuantStudio 7 detection system (Applied Biosystems). Data was expressed as arbitrary units and expression of target genes corrected 
to the geometric average of 3 housekeeping genes: 18S, 36b4 and Tbp (murine data) or 18S, CYCA, GUSB (human data).

\section{NanoString ("Newcastle Cohort")}

Samples were run on the NanoString nCounter analysis system using a custom-made assay panel (Nanostring, Seattle, Washington, USA). Sample input was normalized to 100ng where possible or a maximum of $6 \mu \mathrm{l}$ volume was used. Normalization to housekeeping genes RPL19, SRSF4 and YWHAZ was done using the nSolver 3.0 software (Nanostring).

\section{Whole-transcriptome amplification and RNA sequencing (NGS)}

RNA from tissues (2 ug RNA) or cells (300ng RNA) were used to generate barcoded sequencing libraries using Illumina TruSeq® Stranded mRNA Library Preparation Kit (Illumina) or, for Figure 3 and Extended Data Figure 3, Takara Clontech SMARTer stranded total RNA-Seq Kit v2 - Pico Input Mammalian (Cat \#634412) following manufacturer instructions. Samples failing to show amplification were removed from the experiment and the amplification repeated. The sequencing libraries were normalized for concentration and combined into pools of 96-plex. The pooled libraries were sequenced on 3 lanes of an Illumina HiSeq 4000 instrument at single-end 50bp (SE50), yielding an average of >15 million reads per sample. Library preparation was performed by the Genomics and Transcriptomic Core at the Institute of Metabolic Science. The sequencing was performed at the Genomics Core, Cancer Research UK Cambridge Institute.

\section{Statistics and Reproducibility}

$\mathrm{WD}, \mathrm{PH}$ and CCL4 experiments were performed in 2 batches and the hypothesis that the 2 batches could perform differently was tested with multivariate approaches; this hypothesis was rejected and the data were merged using the whole cohort for the analysis. In vitro experiments were performed at least 2 times with 4 biological replicates (every biological HSC replicate is also a pool of HSC derived from 3 mouse livers). 3D microtissues and CDHFD experiments were performed once. All data are expressed as mean \pm SEM. All analyses were 
performed using NCSS Statistical Package. Statistical significance $(P \leq 0.05)$ was assessed with two sided Student T-test (2 groups), Paired T-test, one-way analysis of variance (ANOVA) plus Fisher's least significant difference (multiple-comparisons), or Multivariate Analysis of Variance (MANOVA), when appropriate. Non-parametric analysis (Kruskal-Wallis one-way analysis of variance on ranks plus Dunn's multiple-comparison test) was used to assess significance of semi-quantitative measures (i.e. histologic scoring) ${ }^{60}$. Data in Table $\mathrm{S} 1$ were retrieved by publicly available databases available on NCBI GEO (https://www.ncbi.nlm.nih.gov/geo/) and analyzed using the online tool "GEO2R". RNA sequencing data was aligned using TopHat V2.0.11 ${ }^{61}$ to the mouse GRCm38 genome or ensembl v92, and genes were counted using HTseq-count ( $v$ 0.8.0). The raw gene-level counts were then used for differential gene expression analysis (two-tailed T-test or GLM likelihood ratio, when appropriate) using LimmaR 3.28.14 and EdgeR 3.8.5.

The raw $p$ values were then adjusted by the Benjamini-Hochberg procedure to control the False Discovery Rate (FDR), i.e. the expected proportion of incorrectly rejected null hypotheses ("false discoveries") ${ }^{62}$.

\section{Bioinformatics functional analyses}

After statistical analysis, differentially expressed genes within groups were studied using the Ingenuity Pathway Analysis (Qiagen). We imputed in IPA the whole transcriptome and then filtered for analysis focused on only statistically significant $(p<0.05)$ items with $-0.378<\log _{2} \mathrm{FC}$ $>$ 0.378. Pathways and "Upstream Regulators" networks showing relationships and interactions experimentally confirmed between differentially expressed genes and others that functionally interact with them, were generated and ranked in terms of significance of participating genes $(p<0.05)$ and activation status $(Z$-score). A comparison analysis was then performed to focus only on those pathways significantly enriched in all the datasets of the same series (Mice: WT - WD vs LFD, WD - KO vs. WT; $\mathrm{CCl}_{4}$ vs. OO; $\mathrm{CCl}_{4}-\mathrm{KO}$ vs. WT. Cells: BMP8 vs. CTRL). We considered "biologically relevant" only those genes that are statistically significant $(p<0.05)$, with a $-0.378<\log _{2} F C>0.378$, and enriched in 'significantly 
Vacca et al, Bmp8b in NASH

modulated' pathways in the comparative analysis, and/or those genes with a FDR $<0.05$. We also performed a "deconvolution analysis" (ImmQuant Software) to for inferring immune celltype composition from gene-expression data using average abundance (Log2CPM) of the whole transcriptome in the different groups tested, analyzing the data against FACS-based and/or signature-based reference data from the ImmGem Consortium ${ }^{63}$.

\section{Data Availability}

The data that support the findings of this study are available from the corresponding authors upon request. In vivo NGS data in this manuscript has been deposited in GEO, accession number GSE110404.

\section{Further details on the methods are available in the Reporting Summary}

\section{METHODS ONLY REFERENCES}

51. Zhao, G.Q., Deng, K., Labosky, P.A., Liaw, L. \& Hogan, B.L. The gene encoding bone morphogenetic protein $8 \mathrm{~B}$ is required for the initiation and maintenance of spermatogenesis in the mouse. Genes Dev 10, 1657-1669 (1996).

52. rodents, F.w.g.o.r.o.g.f.h.m.o., et al. FELASA recommendations for the health monitoring of mouse, rat, hamster, guinea pig and rabbit colonies in breeding and experimental units. Lab Anim 48, 178-192 (2014).

53. Tan, C.Y., et al. Brown Adipose Tissue Thermogenic Capacity Is Regulated by Elovl6. Cell Rep 13, 2039-2047 (2015).

54. Elia, M. \& Livesey, G. Energy expenditure and fuel selection in biological systems: the theory and practice of calculations based on indirect calorimetry and tracer methods. World Rev Nutr Diet 70, 68-131 (1992).

55. Nielsen, M.J., et al. The neo-epitope specific PRO-C3 ELISA measures true formation of type III collagen associated with liver and muscle parameters. Am J Transl Res 5, 303-315 (2013).

56. Barascuk, N., et al. A novel assay for extracellular matrix remodeling associated with liver fibrosis: An enzyme-linked immunosorbent assay (ELISA) for a MMP-9 proteolytically revealed neo-epitope of type III collagen. Clin Biochem 43, 899-904 (2010).

57. Girish, V. \& Vijayalakshmi, A. Affordable image analysis using NIH Image/ImageJ 1.8.0. Indian J Cancer 41, 47 (2004).

58. Wilson, C.L., et al. Ubiquitin C-terminal hydrolase 1: A novel functional marker for liver myofibroblasts and a therapeutic target in chronic liver disease. J Hepatol 63, 1421-1428 (2015).

59. Folch, J., Lees, M. \& Stanley, G.H.S. A Simple Method for the Isolation and Purification of Total Lipides from Animal Tissues. J. Biol. Chem. 226, 497-509 (1957).

60. Gibson-Corley, K.N., Olivier, A.K. \& Meyerholz, D.K. Principles for valid histopathologic scoring in research. Vet Pathol 50, 1007-1015 (2013).

61. Anders, S., Pyl, P.T. \& Huber, W. HTSeq--a Python framework to work with highthroughput sequencing data. Bioinformatics 31, 166-169 (2015).

62. Benjamini, Y., Drai, D., Elmer, G., Kafkafi, N. \& Golani, I. Controlling the false discovery rate in behavior genetics research. Behav Brain Res 125, 279-284 (2001). 
Vacca et al, Bmp8b in NASH

63. Frishberg, A., Brodt, A., Steuerman, Y. \& Gat-Viks, I. ImmQuant: a user-friendly tool for inferring immune cell-type composition from gene-expression data. Bioinformatics 32, 3842-3843 (2016). 